\title{
An ALMA study of the Orion Integral Filament
}

\section{Evidence for narrow fibers in a massive cloud $\star, \star \star$}

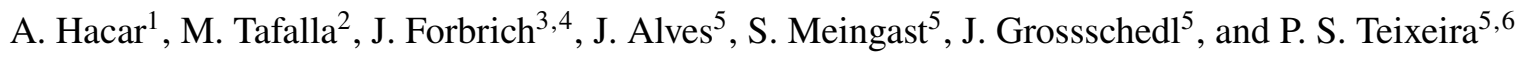 \\ ${ }^{1}$ Leiden Observatory, Leiden University, PO Box 9513, 2300-RA Leiden, The Netherlands \\ e-mail: hacar@strw.leidenuniv.nl \\ 2 Observatorio Astronomico Nacional (IGN), C/Alfonso XII, 3, 28014 Madrid, Spain \\ ${ }^{3}$ Centre for Astrophysics Research, University of Hertfordshire, College Lane, Hatfield AL10 9AB, UK \\ ${ }^{4}$ Harvard-Smithsonian Center for Astrophysics, 60 Garden St, Cambridge, MA 02138, USA \\ ${ }^{5}$ University of Vienna, Türkenschanzstrasse 17, 1180 Vienna, Austria \\ ${ }^{6}$ Scottish Universities Physics Alliance (SUPA), School of Physics and Astronomy, University of St. Andrews, North Haugh, \\ St. Andrews, Fife KY16 9SS, UK
}

Received 4 September 2017 / Accepted 4 January 2018

\begin{abstract}
Aims. We have investigated the gas organization within the paradigmatic Integral Shape Filament (ISF) in Orion in order to decipher whether or not all filaments are bundles of fibers.

Methods. We combined two new ALMA Cycle 3 mosaics with previous IRAM 30m observations to produce a high-dynamic range $\mathrm{N}_{2} \mathrm{H}^{+}$(1-0) emission map of the ISF tracing its high-density material and velocity structure down to scales of $0.009 \mathrm{pc}$ (or $\sim 2000 \mathrm{AU}$ ). Results. From the analysis of the gas kinematics, we identify a total of 55 dense fibers in the central region of the ISF. Independently of their location in the cloud, these fibers are characterized by transonic internal motions, lengths of $\sim 0.15 \mathrm{pc}$, and masses per unit length close to those expected in hydrostatic equilibrium. The ISF fibers are spatially organized forming a dense bundle with multiple hublike associations likely shaped by the local gravitational potential. Within this complex network, the ISF fibers show a compact radial emission profile with a median FWHM of $0.035 \mathrm{pc}$ systematically narrower than the previously proposed universal 0.1 pc filament width.

Conclusions. Our ALMA observations reveal complex bundles of fibers in the ISF, suggesting strong similarities between the internal substructure of this massive filament and previously studied lower-mass objects. The fibers show identical dynamic properties in both low- and high-mass regions, and their widespread detection in nearby clouds suggests a preferred organizational mechanism of gas in which the physical fiber dimensions (width and length) are self-regulated depending on their intrinsic gas density. Combining these results with previous works in Musca, Taurus, and Perseus, we identify a systematic increase of the surface density of fibers as a function of the total mass per-unit-length in filamentary clouds. Based on this empirical correlation, we propose a unified starformation scenario where the observed differences between low- and high-mass clouds, and the origin of clusters, emerge naturally from the initial concentration of fibers.
\end{abstract}

Key words. ISM: clouds - ISM: kinematics and dynamics - ISM: structure - stars: formation - submillimeter: ISM

\section{Introduction}

Investigating the internal structure of massive filaments is of crucial importance for the description of the star formation process in the Milky Way. As indicated by recent galactic plane surveys, high-mass stars and massive clusters are typically formed within filaments with total masses per-unit-length $\left(\mathrm{M}_{\text {lin }}\right)$ between $\sim 100$ and $4000 M_{\odot} \mathrm{pc}^{-1}$ (e.g., Peretto \& Fuller 2009; Molinari et al. 2010; Schisano et al. 2014; Li et al. 2016). Typically located at kpc distances, most of these massive filaments are found in hub-like associations (Myers 2009) forming dense ridges of gas (Galván-Madrid et al. 2010; Schneider et al. 2010; Henning et al. 2010; Hill et al. 2011; Hennemann et al. 2012; Peretto et al. 2013). Morphological and dynamical arguments suggest a direct link between these massive filaments and their better

\footnotetext{
* The movie associated to Fig. 2 is available at http://www . aanda.org

$\star \star$ The data products of this work are only available at the CDS via anonymous ftp to cdsarc.u-strasbg. fr (130.79.128.5) or via http://cdsarc.u-strasbg.fr/viz-bin/qcat?]/A+A/610/A77
}

characterized low-mass counterparts with $\mathrm{M}_{\text {lin }} \lesssim 100 M_{\odot} \mathrm{pc}^{-1}$, regularly identified in the solar neighborhood (André et al. 2010; Arzoumanian et al. 2011; Hacar \& Tafalla 2011; Hacar et al. 2013; Palmeirim et al. 2013; Hacar et al. 2016). So far, however, a detailed comparison between these two filamentary regimes has been hampered by the resolution and sensitivity of current far-infrared (FIR) and (sub-)millimeter observations. As a result, the connection between low- and high-mass filaments remains controversial (e.g., see André et al. 2014; Motte et al. 2017).

Recent molecular observations have revealed the intrinsic substructure of low-mass filaments in nearby clouds. Hacar et al. (2013) demonstrated that the apparently monolithic B213-L1495 filament $\left(\mathrm{M}_{\text {lin }} \sim 50 \mathrm{M}_{\odot} \mathrm{pc}^{-1}\right.$; Barnard et al. 1927; Hartmann 2002; Palmeirim et al. 2013) is actually a bundle of smallscale fibers. These fibers are characterized by their continuity in space and velocity, transonic internal velocity dispersions along their typical length of $\sim 0.5 \mathrm{pc}$, and individual $\mathrm{M}_{\text {lin }}$ consistent with hydrostatic equilibrium. After this discovery, analogous fibers have been systematically reported in low-mass filaments $\left(\mathrm{M}_{\text {lin }} \sim 20-50 \mathrm{M}_{\odot} \mathrm{pc}^{-1}\right.$ ) like IC5146 (Arzoumanian et al. 2013), 
Musca (Hacar et al. 2016), and TMC-1 (Fehér et al. 2016). Compact networks of fibers have also been identified in the NGC1333 ridge $\left(\mathrm{M}_{\text {lin }} \sim 200 M_{\odot} \mathrm{pc}^{-1}\right.$; Hacar et al. 2017b). In all cases, the fibers harbor most of the cores within these regions, regulating the initial conditions for their gravitational collapse (see also Hacar \& Tafalla 2011; Tafalla \& Hacar 2015). Dominating the gas substructure in isolated and clustered environments, fibers appear to play a fundamental role in both low- and intermediate mass filaments.

In analogy to low-mass filaments, high-mass filaments, such as Nessie $\left(\mathrm{M}_{\text {lin }} \sim 525 M_{\odot} \mathrm{pc}^{-1}\right.$; Jackson et al. 2010), G11.1 $\left(\mathrm{M}_{\text {lin }} \sim 600 M_{\odot} \mathrm{pc}^{-1}\right.$; Kainulainen et al. 2013), and NGC6334 $\left(\mathrm{M}_{\text {lin }} \sim 1000 M_{\odot} \mathrm{pc}^{-1}\right.$; André et al. 2016) exhibit an increasing level of substructure at sub-parsec scales. Additional observational evidence indicates the existence of fibers in some of these massive environments. Complex line profiles containing multiple narrow velocity components are commonly reported towards Infrared Dark Clouds (IRDC) like G035 $\left(\mathrm{M}_{\text {lin }} \sim 100 M_{\odot} \mathrm{pc}^{-1}\right.$; Henshaw et al. 2014), G14.225 ( $\mathrm{M}_{\text {lin }} \sim 200 M_{\odot} \mathrm{pc}^{-1}$; Busquet et al. 2013), or IRDC $18223\left(\mathrm{M}_{l i n} \sim 1000 M_{\odot} \mathrm{pc}^{-1}\right.$; Beuther et al. 2015). These velocity components appear to be organized in elongated, sub-parsec scale, fiber-like threads partially resolved in recent interferometric observations (Henshaw et al. 2016, 2017). In light of the above, can fibers also explain the internal structure of these massive clouds?

In this work (Paper I) we investigate the dense gas substructure of the paradigmatic Orion Integral Shape Filament (ISF; Bally et al. 1987) combining a new set of ALMA Cycle-3 with IRAM 30m observations. Along its more than $7 \mathrm{pc}$ of length, the ISF describes a dense ridge of gas of $\lesssim 0.2 \mathrm{pc}$ width, running approximately parallel to the declination axis at the northern end of the Orion A cloud (Johnstone \& Bally 1999). The ISF is the most massive filament among the Gould Belt clouds $\left(\mathrm{M}_{\text {lin }} \sim 500 \mathrm{M}_{\odot} \mathrm{pc}^{-1}\right.$; Bally et al. 1987) and the only one containing a high-mass cluster, namely, the Orion Nebula Cluster (ONC; O'Dell et al. 2008). Due to its proximity ( $D=414 \mathrm{pc}$; Menten et al. 2007), the ISF is one of the best-studied massive filaments and is usually employed as benchmark for clustered star-formation theories (see Bally 2008; Muench et al. 2008, and references therein).

At large scales, the gas content of the ISF has been systematically surveyed using single-dish observations of multiple CO isotopologs (Bally et al. 1987; Dutrey et al. 1991; Shimajiri et al. 2011; Berné et al. 2014; Buckle et al. 2012; Shimajiri et al. 2014), dense tracers (Ikeda et al. 2007; Tatematsu et al. 2008; Hacar et al. 2017a; Friesen et al. 2017; Kauffmann et al. 2017), and recombination lines (Goicoechea et al. 2015). Its mass distribution has also been investigated in the continuum at both (sub-)millimeter (Chini et al. 1997; Johnstone \& Bally 1999; Salji et al. 2015) and FIR wavelengths (Lombardi et al. 2014; Stutz \& Kainulainen 2015) showing a series of clumps regularly spaced at scales of $\sim 1$ pc (Dutrey et al. 1991), typically referred to as the OMC 1-4 clouds (see also Peterson $\&$ Megeath 2008). Higher-resolution studies reveal a rich substructure of small-scale sub-filaments (Martin-Pintado et al. 1990; Rodriguez-Franco et al. 1992; Wiseman \& Ho 1998; Li et al. 2013; Hacar et al. 2017a) and condensations (Mezger et al. 1990; Chini et al. 1997) extending along the main axis of the ISF. A census of its embedded stellar population at IR (Megeath et al. 2012; Stutz et al. 2013; Furlan et al. 2016), X-ray (Getman et al. 2005; Rivilla et al. 2013), centimeter (Kounkel et al. 2014; Forbrich et al. 2016), and millimeter (Takahashi et al. 2013; Teixeira et al. 2016; Kainulainen et al. 2017; Palau et al. 2017) wavelengths indicate that most of the current star formation within the ISF is concentrated towards both OMC1 and OMC-2/3 clouds (Peterson \& Megeath 2008). Focused on these two active subregions, our new $\mathrm{N}_{2} \mathrm{H}^{+}$(1-0) ALMA observations (Sect. 2) aim to explore the existence of fibers within this massive filament (Sect. 3), as well as their possible connection with the formation of massive stars and clusters (Sect. 4).

\section{ALMA Cycle-3 observations}

We mapped the central region of the ISF between December 26, 2015, and January 2, 2016, using ALMA Cycle-3 observations (ID: 2015.1.00669.S; PI: A. Hacar) ${ }^{1}$. As shown in Fig. 1, we combined two 148-pointing ALMA mosaics, of $240^{\prime \prime} \times 600^{\prime \prime}$ each, following the main axis of this cloud traced by previous single-dish observations (Hacar et al. 2017a). The first of these mosaics targeted the OMC-1 region (blue footprints in Fig. 1, right) covering the central region of the ONC, including the Trapezium and the Orion BN source, the Orion BN/KL explosion (Bally et al. 2011), the OMC-1 South proto-cluster (Grosso et al. 2005), the OMC-1 ridge (Wiseman \& Ho 1998, also referred to as $\mathrm{OMC}-1 \mathrm{~N})$, and some of most prominent dense molecular fingers (Rodriguez-Franco et al. 1992). Continuing to the north, the second mosaic mapped the OMC-2 and the southern end of the OMC-3 regions (red footprints in Fig. 1, right) covering all the previously identified FIR sources (OMC-2 FIR 1-6; Mezger et al. 1990) and several of the millimeter sources (MMS 8-10; Chini et al. 1997) within these two clouds.

As primary target line of this project, we observed the $\mathrm{N}_{2} \mathrm{H}^{+}$(1-0) line emission in Band 3 (93173.764 MHz; Pagani et al. 2009) at high spectral resolution $\left(30 \mathrm{kHz}\right.$ or $\left.0.1 \mathrm{~km} \mathrm{~s}^{-1}\right)$. Three additional broad-band, $1.8 \mathrm{GHz}$ wide spectral windows were observed simultaneously covering the continuum centered at $~ 93,104$, and $106 \mathrm{GHz}$. The observations were carried out with $\mathrm{PWV}=2-5 \mathrm{~mm}$ and in the most compact configuration of the ALMA $12 \mathrm{~m}$ array (C36-1 and C36-2) with baselines between 15.1 and $310 \mathrm{~m}$. The quasar J0423-0120 was observed for bandpass plus amplitude calibrations at the beginning of each observing block. Phase calibration was performed on J0541-0541 every $\sim 10 \mathrm{~min}$. Independent visibility data for each mosaic were obtained in CASA (v4.5.3) (McMullin et al. 2007) using the facility provided pipeline.

Our two $\mathrm{N}_{2} \mathrm{H}^{+}$ALMA mosaics were simultaneously imaged in CASA (v4.7.1) in combination with previous single-dish IRAM 30m observations of the ISF $\left(\theta_{F W H M}=3^{\prime \prime}\right.$; Hacar et al. 2017a) using standard techniques. First, we subtracted the continuum emission from our high-resolution spectral window based on the line-free continuum level estimated in all sidebands. Second, the line visibilities were simultaneously deconvolved with the CASA task clean using the single-dish observations as source model and a Briggs weighting with robust parameter equal to 0.5 . Third, the resulting primary-beam corrected image (at $\sim 3.5^{\prime \prime} \times 3.0^{\prime \prime}$ resolution) was convolved into a final circular beam $\left(\theta_{F W H M}\right)$ of $4.5^{\prime \prime}$ in order to improve the sensitivity and stability of our maps. Finally, both single-dish and interferometric maps were combined using the task feathering in order to maximize the recovery of the extended emission filtered by the $12 \mathrm{~m}$ array. The rms level of our final map, estimated from the analysis of line-free channels, is $25 \mathrm{mJy} \mathrm{beam}^{-1}$ at

1 This work is part of the ORION-4D project (PI: A. Hacar). See more information in https://sites.google.com/site/ orion4dproject 

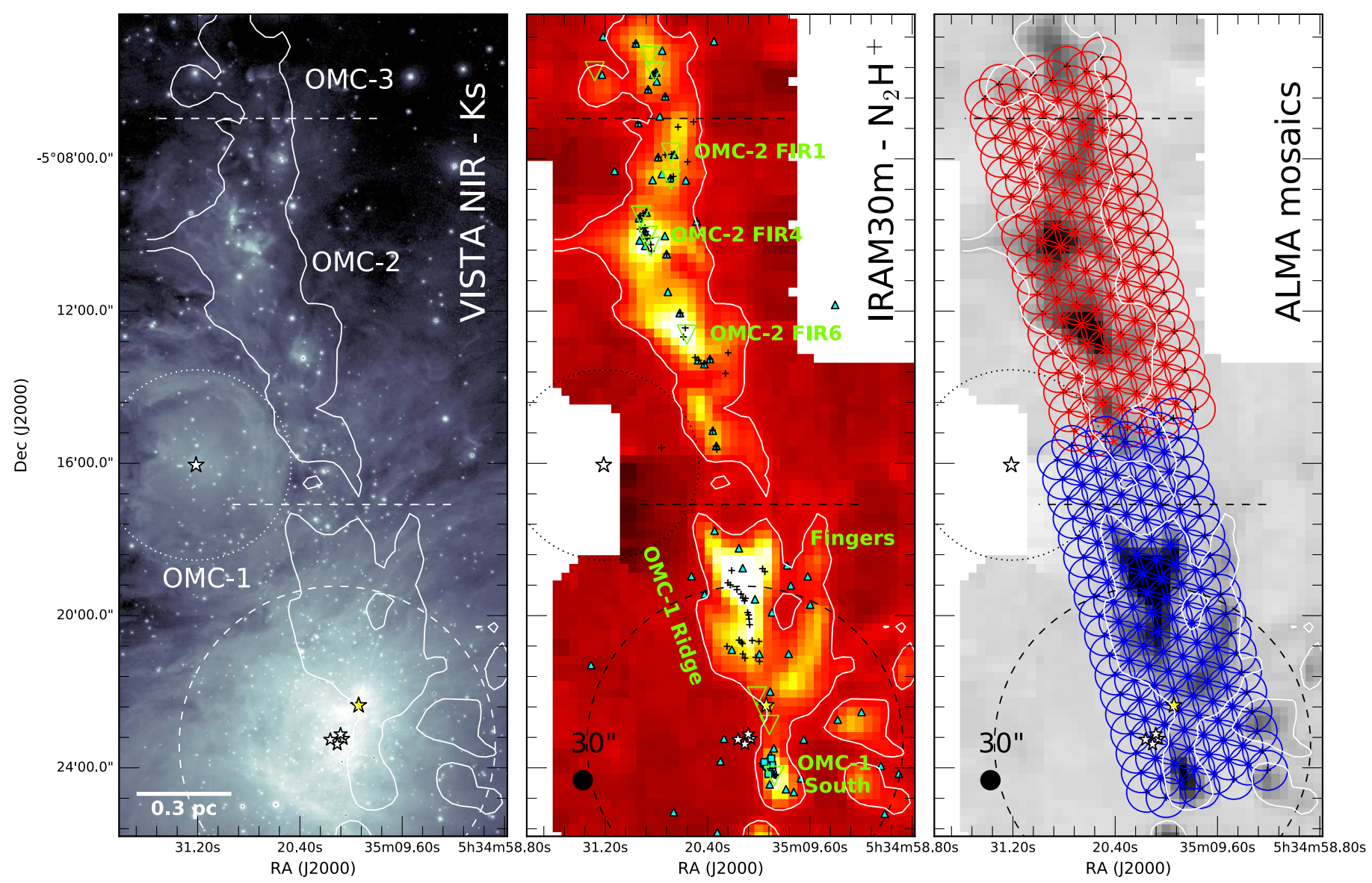

Fig. 1. Description of our ALMA Cycle-3 observations along the ISF: left: VISTA NIR ( $K s$ band) emission (Meingast et al. 2016); center: IRAM 30m (single-dish) $\mathrm{N}_{2} \mathrm{H}^{+}$(1-0) integrated emission (Hacar et al. 2017a); right: $12 \mathrm{~m}$-array footprints of the two ALMA Cycle-3 mosaics (blue and red) presented in this work. The position of the Trapezium (white stars) and NU Ori stars (white isolated star), the Orion BN source (yellow star), and the innermost $0.5 \mathrm{pc}$ radius of the ONC (dashed circle) are indicated in all panels. All figures include the contour enclosing those regions with integrated emission $\mathrm{I}_{2}\left(\mathrm{~N}_{2} \mathrm{H}^{+}\right) \geq 20 \mathrm{mJy}$ beam ${ }^{-1}$ according to the single-dish observations. The position of all the previously identified Spitzer protostars (blue triangles; Megeath et al. 2012; Stutz et al. 2013; Furlan et al. 2016), mm-continuum peaks (green triangles; Mezger et al. 1990; Chini et al. 1997), SMA (Teixeira et al. 2016) plus ALMA continuum sources (Kainulainen et al. 2017; Palau et al. 2017) (black crosses), and embedded X-ray objects (blue squares; Rivilla et al. 2013) are indicated in the central panel. The most relevent regions are also labelled in both VISTA and single-dish images. For reference, a scale bar denotes the angular size of a $0.3 \mathrm{pc}$ region at the distance of the ONC (414 pc; Menten et al. 2007).

a spectral resolution of $0.1 \mathrm{~km} \mathrm{~s}^{-1}$. Assuming a flux conversion factor of $13.6\left(\frac{300 \mathrm{GHz}}{v}\right)^{2}\left(\frac{1^{\prime \prime}}{\Theta_{F W H M}}\right)^{2}=6.96 \mathrm{~K} \mathrm{Jy}^{-1}$ (ALMA technical handbook), the above estimates translate into a brightness temperature sensitivity of $0.17 \mathrm{~K}$ in main beam units. The recovered signal in our spectra covers a wide dynamic range in intensities with peak values up to a signal-to-noise ratio (S/N) $\gtrsim 50$ with respect to the noise levels in both total integrated emission and individual line intensities. A detailed discussion on the data-reduction process will be presented in a subsequent paper (Paper II; Hacar et al., in prep.).

\section{Results}

We investigated the internal gas substructure of the ISF from the analysis of the $\mathrm{N}_{2} \mathrm{H}^{+}$(1-0) line emission. Enhanced as result of the $\mathrm{CO}$ freeze-out, $\mathrm{N}_{2} \mathrm{H}^{+}$is an ideal tracer of cold gas with densities of $n\left(\mathrm{H}_{2}\right) \gtrsim 5 \times 10^{4} \mathrm{~cm}^{-3}$ (Bergin \& Tafalla 2007). The emission of its ground transition $\mathrm{J}=(1-0)$ has been traditionally employed in observations of dense cores (e.g., Caselli et al. 2002). Recent studies of massive star-forming regions indicate that the emission of this tracer is not restricted to these stellar embryos but that it extends to large scales in dense environments (Fernández-López et al. 2014; Henshaw et al. 2016; Hacar et al. $2017 b$ ). In the case of the ISF, single-dish observations indicate a widespread and intense $\mathrm{N}_{2} \mathrm{H}^{+}$emission along the main axis of this massive filament (Tatematsu et al. 2008; Hacar et al. 2017a).

On the other hand, $\mathrm{N}_{2} \mathrm{H}^{+}$also presents several observational advantages for star-formation studies. Unlike the FIR/mm continuum observations (e.g., Herschel) sensitive to the total column density, $\mathrm{N}_{2} \mathrm{H}^{+}$selectively highlights the high-density, star-forming material in compact regions (e.g., Pety et al. 2016; Hacar et al. 2017b). Moreover, its emission properties and hyperfine structure enable high accuracy studies of the gas kinematics otherwise hampered by the more complex hyperfine structure in other dense tracers like ammonia (see a discussion in Hacar et al. 2017b).

Figure 2 (right) shows the total integrated intensity $\mathrm{N}_{2} \mathrm{H}^{+}$(1-0) emission map obtained after the combination of our IRAM 30m and ALMA observations. The resulting ALMA mosaic covers an approximate area of $2.5 \times 0.48 \mathrm{pc}^{2}$ with an effective resolution of 0.009 pc (i.e., $\theta_{F W H M}=4.5^{\prime \prime}$ at the distance of 414 pc; Menten et al. 2007). Several prominent gas concentrations are coincident with the position of the 


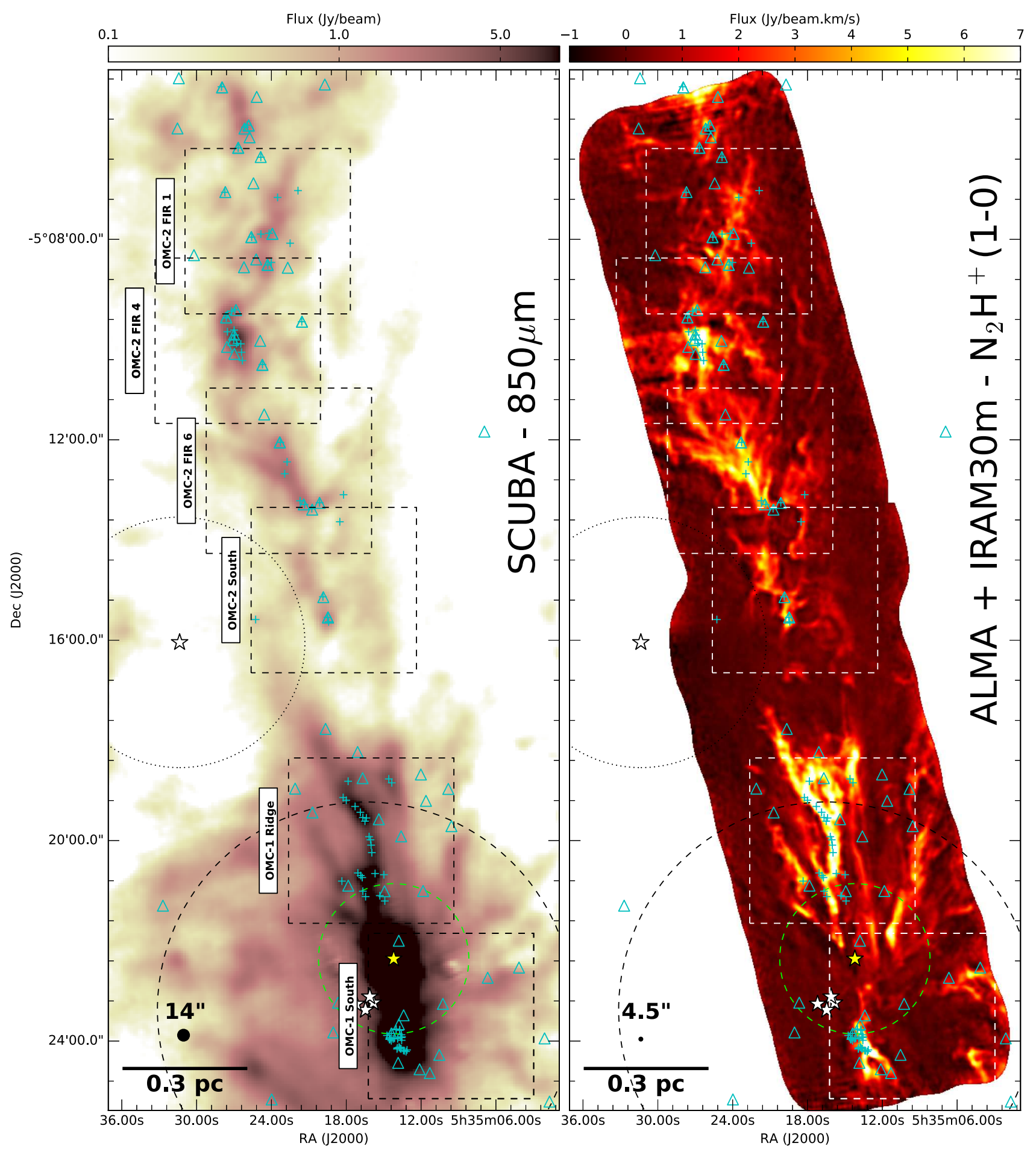

Fig. 2. Dense gas distribution within the ISF. Left: SCUBA- $850 \mu \mathrm{m}$ continuum emission (Johnstone \& Bally 1999). Right: total $\mathrm{N}_{2} \mathrm{H}^{+}$integrated intensity mosaic obtained by the combination of ALMA $12 \mathrm{~m}$ and IRAM $30 \mathrm{~m}$ data. For reference, the positions of the Trapezium (white stars in OMC-1), the Orion BN source (yellow stars), the size of the Orion BN/KL explosion (green dashed circle; Bally et al. 2011), the 0.5 pc radius of the ONC (dashed line), the extension of the M43 nebula (dotted circle; Subrahmanyan et al. 2001) powered by NU Ori (isolated white star), and both Spitzer protostars (blue triangles; Megeath et al. 2012; Stutz et al. 2013; Furlan et al. 2016) plus continuum sources (blue crosses; Teixeira et al. 2016; Kainulainen et al. 2017; Palau et al. 2017) are shown in both panels. The corresponding beamsize (black solid dot) is indicated in the lower left corner in comparison with a characteristic $0.3 \mathrm{pc}$ scale (black bar). The position of the zoom-in regions presented in Fig. 3 are enclosed by dashed boxes. A movie showing the combined ALMA $12 \mathrm{~m}$ plus IRAM 30m mosaic is available online.

OMC-1 South proto-cluster, the OMC-1 Ridge region, and the OMC-2 FIR 1, FIR 4, plus FIR 6 sources with flux densities $>3 \mathrm{Jy} \mathrm{beam}^{-1} \mathrm{~km} \mathrm{~s}^{-1}$. In addition, a rich filamentary substructure can be identified at lower intensities. Among them, the well-known molecular fingers in the OMC-1 region (RodriguezFranco et al. 1992) are clearly seen in these observations.
Our new ALMA mosaic allows us to investigate the distribution of dense and star-forming gas in the ISF with unprecedented detail. As illustrated in Fig. 2, we find a close correspondence between the recovered total $\mathrm{N}_{2} \mathrm{H}^{+}$intensity and the high- column-density material traced in previous $850 \mu \mathrm{m}$-SCUBA observations (Johnstone \& Bally 1999, 

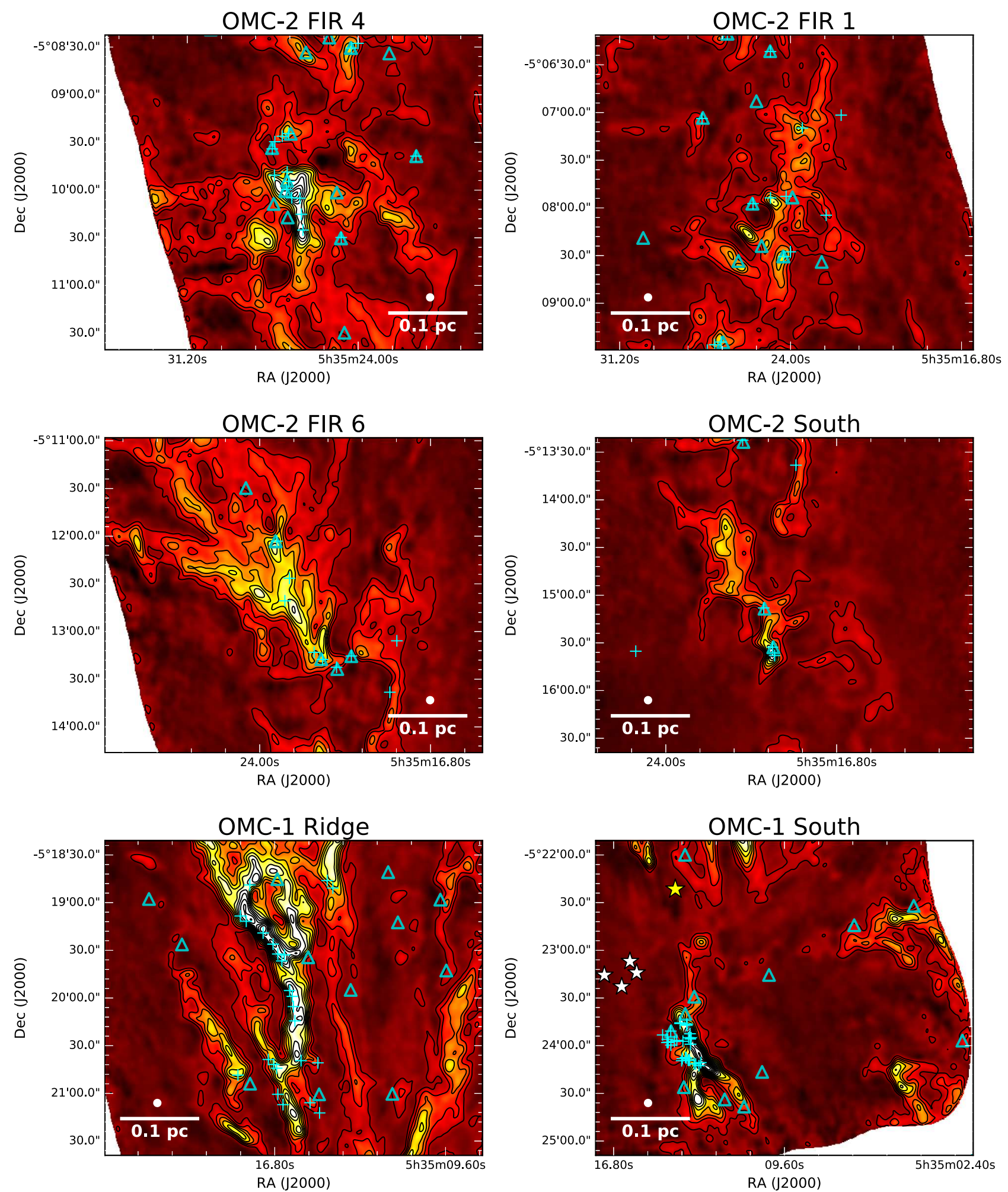

Fig. 3. Close-up view of the total $\mathrm{N}_{2} \mathrm{H}^{+}$integrated emission in six selected regions along the ISF (see also Fig. 2). From left to right and from top to bottom: (a) OMC-2 FIR 4; (b) OMC-2 FIR 1; (c) OMC-2 FIR 6; (d) OMC-2 South; (e) OMC-1 Ridge; and (f) OMC-1 South. The positions of all the Spitzer protostars (blue triangles; Megeath et al. 2012; Stutz et al. 2013; Furlan et al. 2016) and continuum sources (blue crosses; Teixeira et al. 2016; Kainulainen et al. 2017; Palau et al. 2017) are indicated in all subpanels. Contours are equally spaced every $1 \mathrm{Jy} \mathrm{beam}^{-1} \mathrm{~km} \mathrm{~s}^{-1}$. The beamsize (white solid dot) and the characteristic $0.1 \mathrm{pc}$ scale (white bar) are indicated in the lower corner of each subpanel.

$\left.\theta_{F W H M}=14.0^{\prime \prime}\right)$. Similarly, the $\mathrm{N}_{2} \mathrm{H}^{+}$emission features mimic the dense gas distribution reported in interferometric $\mathrm{VLA}-\mathrm{NH}_{3}$ maps of OMC-1 (Wiseman \& Ho 1998, $\theta_{F W H M}=8.5^{\prime \prime} \times 9^{\prime \prime}$, $\delta v=0.3 \mathrm{~km} \mathrm{~s}^{-1}$ ) and OMC-2 (Li et al. 2013, $\theta_{F W H M}=5^{\prime \prime}$, $\delta v=0.6 \mathrm{~km} \mathrm{~s}^{-1}$ ). With the only exception of the hot gas component at the vicinity of the Orion $\mathrm{BN} / \mathrm{KL}$ region $\left(\mathrm{T}_{K}>100 \mathrm{~K}\right.$;
Genzel et al. 1982; Goddi et al. 2011), $\mathrm{N}_{2} \mathrm{H}^{+}$accurately traces the dense and cold material $\left(\mathrm{T}_{K} \lesssim 30 \mathrm{~K}\right)$ in this cloud. The $\mathrm{N}_{2} \mathrm{H}^{+}$integrated emission also encloses $>90 \%$ of the Spitzer protostars (blue triangles; Megeath et al. 2012; Stutz et al. 2013; Furlan et al. 2016) and compact continuum sources (blue crosses; Kainulainen et al. 2016; Teixeira et al. 2016; Palau et al. 2017). 
In addition to these previous works, the improved spatial and spectral resolutions of our ALMA observations $\left(\theta_{F W H M}=4.5^{\prime \prime}\right.$, $\delta v=0.1 \mathrm{~km} \mathrm{~s}^{-1}$ ) enable an accurate characterization of the dense gas properties both in space and velocity.

The high dynamic range of our $\mathrm{N}_{2} \mathrm{H}^{+}$ALMA + IRAM 30m observations reveals the intricate gas substructure of the massive ISF. As highlighted in Fig. 3 (see boxes), the most prominent clumps identified in previous studies split into multiple independent elongated substructures at sub-parsec scales, clearly separated at the resolution of our ALMA observations (e.g., see OMC-2 South or OMC-2 FIR-1). Each of these substructures branches off into smaller filamentary features below $0.1 \mathrm{pc}$. At smaller scales, these latter objects seem to fragment, forming series of prolate condensations with major axes between $\sim 0.01$ and $0.03 \mathrm{pc}$. Despite their differences in terms of star-formation activity and feedback, the same gas organization is simultaneously observed in both OMC-1 and OMC-2 regions (see also Sect. 3.5). Within the boundaries of our maps, this substructure extends over (at least) two orders of magnitude in scale between 0.02 and $\sim 2$ pc.

The above hierarchical organization of filaments within filaments resembles the so-called bundles of fibers identified in low-mass filaments (Hacar et al. 2013, 2016) and intermediatemass clusters (Fernández-López et al. 2014; Hacar et al. 2017b). Our ALMA observations demonstrate that the existence of these fiber networks is not restricted to low-mass regions but extends to filaments at higher-mass regimes like the ISF. In the following subsections we investigate the main physical properties of these new ISF fibers in more detail.

\subsection{Fibrous substructure of the ISF}

We have characterized the internal gas substructure of the ISF using a new version of the FIVE analysis technique (Hacar et al. 2013), hereafter referred to as HiFIVE. A description of the algorithm and its performance can be found in Appendix A. In brief, HiFIVE uses a new hierarchical scheme to systematically identify and characterize velocity-coherent structures in complex molecular line datasets with large dynamic range and highly variable velocity fields. HiFIVE carries out this analysis from the continuity of the gas velocity centroids both in space and velocity using a linking velocity gradient adapted to the local line properties. We analyzed more than 70000 spectra in our data to find $\sim 25000$ components with $S / N \geq 3$ (Table 1; see also Appendix B). Using HiFIVE, we identify a total of 55 velocitycoherent elongated fibers along the ISF. Our results reveal the extraordinary fibrous nature of the dense gas within this massive filament. Still partially unresolved in our HiFIVE analysis, these 55 fibers should be interpreted as a first-order description of the real dense gas substructure in the ISF (see Appendix A for a discussion).

We have defined the main axis of each individual fiber (red segments in Fig. 4) using the same fitting procedure introduced in Hacar et al. (2013). Reinforcing the similarities with previous studies, many of the ISF fibers appear to be well isolated in space and can be recognized in the integrated intensity maps showing large aspect ratios (e.g., fibers \# 25 or 41 ). In Table 2, we summarize the average fiber properties calculated along their main axes. Also displayed in Fig. 5 (left), the ISF fibers present a wellconstrained distribution of sizes with an average total length of $0.16 \pm 0.10 \mathrm{pc}$ (orange filled histogram) without correcting for projection effects.

The observed fiber substructure accurately reproduces the internal dense gas distribution of the ISF. Among the total
$295 M_{\odot}$ of dense gas detected in our high-S/N $\mathrm{N}_{2} \mathrm{H}^{+}$spectra, $288 M_{\odot}$ (i.e. 98\%) are recovered as fibers (see the conversion between the total $\mathrm{N}_{2} \mathrm{H}^{+}$integrated intensities and total column densities in Sect. 3.3). In most cases, we notice a correspondence between these $\mathrm{N}_{2} \mathrm{H}^{+}$fibers and the intensity enhancements detected in the continuum (see Fig. 4, right panel). Indeed, the vast majority of compact sources and protostars in the ISF are found in association to these fibers (e.g., fibers \# 21, 37, 43, etc.). This complex fiber distribution entirely determines the internal organization of the ISF.

Overall, we find no correlation between the orientation of fibers and large-scale feedback effects in the ISF (see also Sect. 3.5). The fibers are distributed irrespective of the stellar activity within the cloud (e.g., see ONC vs. OMC-2 FIR 1). While originally independent, some of the fibers might still be locally influenced by the presence of stars. Within the OMC-1 region, fibers are radially oriented pointing towards the OMC-1 South proto-cluster, likely reflecting the global gravitational collapse of this cloud (Hacar et al. 2017a, see also Appendix B.2). The extension of these fibers beyond the Orion BN/KL explosion (green dashed circle in Fig. 2, Bally et al. 2011) rules out a direct connection with this energetic event. In some specific cases, however, the individual fiber morphology might be potentially altered by the local influence of stars (e.g., fibers \#25 \& \#37), and both the M43 nebula (e.g., fibers \#30 \& \#34) or the ONC (e.g., fibers \#19 \& 24) (see also Fig. 2). In spite of these localized effects, the reported fiber organization appears to reflect the original gas substructure before the formation of stars.

\subsection{Kinematic properties: subsonic fibers in massive clouds}

Investigating the magnitude of the line-of-sight (l.o.s.) nonthermal velocity dispersion $\sigma_{N T}$ is of fundamental importance to characterize the internal dynamical state of fibers (e.g., Hacar $\&$ Tafalla 2011). This observable can be estimated from the measured line full-width-at-half-maximum $(\Delta V)$ obtained from our hyperfine fits (see Appendix A):

$\sigma_{N T}=\left[\left(\frac{\Delta V}{\sqrt{8 \ln 2}}\right)^{2}-\frac{k_{\mathrm{B}} T_{\mathrm{K}}}{\mu\left(\mathrm{N}_{2} \mathrm{H}^{+}\right)}\right]^{1 / 2}$.

Compared to the (local) thermal sound speed for $\mathrm{H}_{2}$, $\mathrm{c}_{s}\left(T_{\mathrm{K}}\right)=\sqrt{\frac{k_{\mathrm{B}} T_{\mathrm{K}}}{\mu\left(\mathrm{H}_{2}\right)}}$, the ratio $\sigma_{N T} / \mathrm{c}_{s}\left(T_{\mathrm{K}}\right)$ can be used as a diagnostic tool to determine whether the observed gas motions are subsonic $\left(\sigma_{N T} / \mathrm{c}_{s}\left(T_{\mathrm{K}}\right) \leq 1\right)$, transonic $\left(1<\sigma_{N T} / \mathrm{c}_{s}\left(T_{\mathrm{K}}\right) \leq 2\right)$, or supersonic $\left(\sigma_{N T} / \mathrm{c}_{s}\left(T_{\mathrm{K}}\right)>2\right)$.

In the absence of strong feedback effects, the dense gas in clouds is found at low and relatively uniform temperatures, typically at $T_{\mathrm{K}} \sim 10 \mathrm{~K}$ (Myers \& Benson 1983). Based on this property, the dynamical state of fibers in quiescent environments is commonly evaluated assuming a single $\mathrm{H}_{2}$ sound speed $\mathrm{c}_{s}\left(T_{\mathrm{K}}\right) \sim \mathrm{c}_{s}(10 \mathrm{~K})$ (e.g., Hacar et al. 2013, 2017b). Contrary to low-mass clouds, the $\mathrm{NH}_{3}$-derived $T_{\mathrm{K}}$ values in the vicinity of the ONC indicate large thermal variations leading to $\mathrm{c}_{s}\left(T_{\mathrm{K}}\right) \gg$ $\mathrm{c}_{s}(10 \mathrm{~K})$ (e.g., see Wiseman \& Ho 1998). To correctly evaluate these thermal effects, we combined our new ALMA observations with the $\mathrm{NH}_{3} \mathrm{~T}_{K}$ estimates provided by the GAS-DR1 survey $\left(\theta_{F W H M}=32^{\prime \prime}\right.$; see details in Friesen et al. 2017). As firstorder approximation, we adopt the $\mathrm{T}_{K}$ value for each individual $\mathrm{N}_{2} \mathrm{H}^{+}$component from the nearest position surveyed in $\mathrm{NH}_{3}{ }^{2}$.

2 We note that the GAS-NH${ }_{3}$ survey provides a unique temperature per position. When multiple $\mathrm{N}_{2} \mathrm{H}^{+}$components are identified in a single ALMA spectrum, the same $T_{K}$ value is assigned to all of them. 
A. Hacar et al. : An ALMA study of the Orion Integral Filament. I.

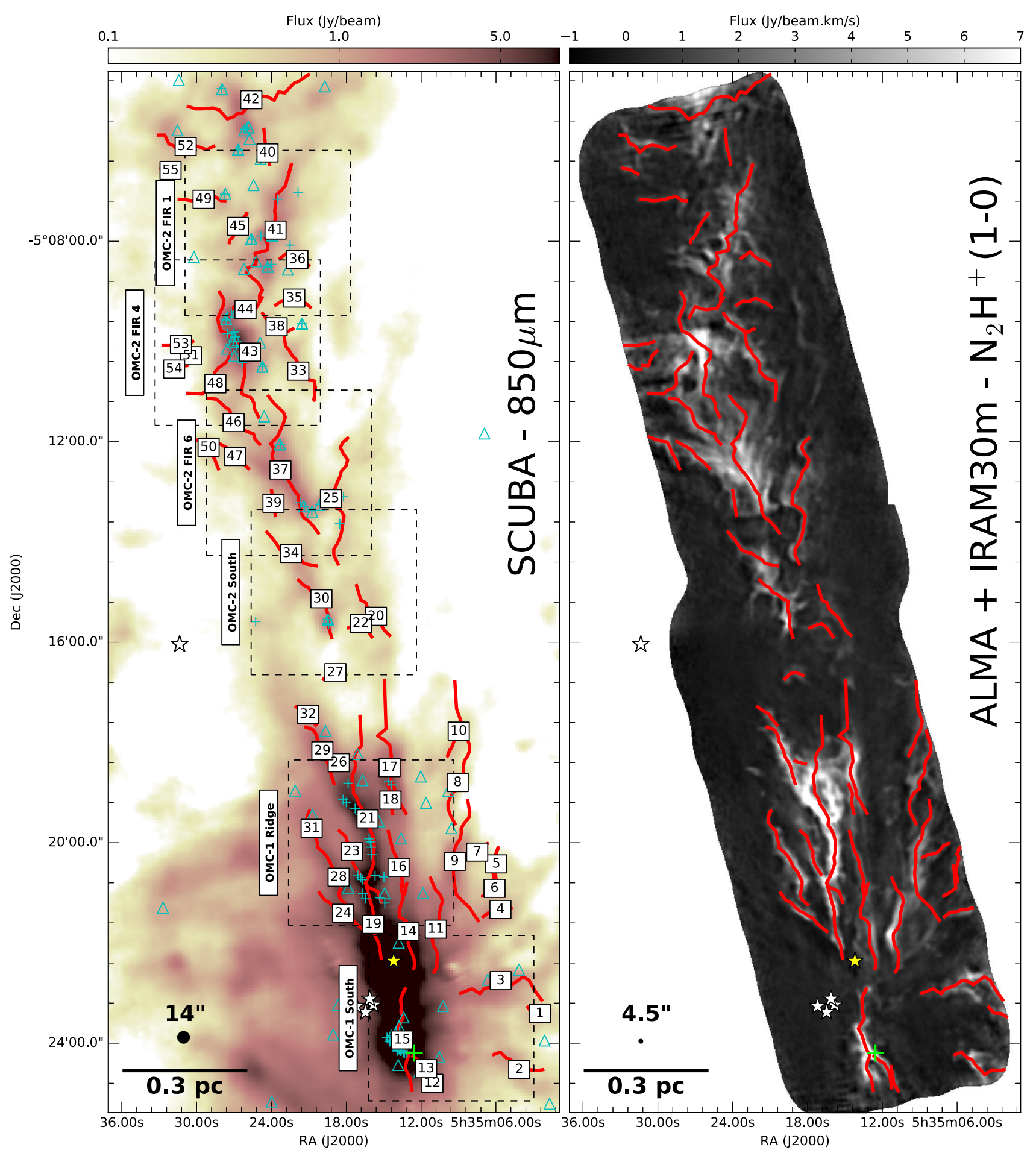

Fig. 4. Main axis of the 55 fibers identified by our HiFIVE analysis along the ISF (red segments). Left: SCUBA-850 $\mu$ m continuum emission (Johnstone \& Bally 1999). Right: total $\mathrm{N}_{2} \mathrm{H}^{+}$integrated emission. The position of both protostars (blue triangles) and continuum sources (blue crosses) are indicated similar to Fig. 2. For reference, the positions of the Trapezium and NU Ori stars (white stars) plus the Orion BN source (yellow stars), are highlighted in both panels. The corresponding beamsizes are indicated in the lower left corner in comparison with a characteristic $0.3 \mathrm{pc}$ scale (black bar). The position of the zoom-in regions presented in Fig. 3 are enclosed by dashed boxes in the SCUBA map. We notice the good correspondence between the position of fibers and the location of the intensity enhancements in the continuum. A green cross indicates the positions with the most blue-shifted velocity detected in our $\mathrm{N}_{2} \mathrm{H}^{+}$maps (see also Appendix B.2), adopted as the approximate center of collapse of the OMC-1 region (Hacar et al. 2017a).

With similar density regimes traced by these twin molecules (see Hacar et al. 2017b), these $\mathrm{NH}_{3}$-derived temperatures provide good estimates for the thermal state of the dense gas detected in $\mathrm{N}_{2} \mathrm{H}^{+}$. In Table 1 we list the average $T_{\mathrm{K}}$ estimates and their corresponding sound speed $\mathrm{c}_{s}\left(T_{\mathrm{K}}\right)$ values in our maps.
We summarize the statistical properties of the derived $\sigma_{N T}$ measurements obtained using Eq. (1) for all the $\mathrm{N}_{2} \mathrm{H}^{+}$ components detected with $S / N \geq 3$ in Table 1 . This table also includes the fraction of components in different turbulent regimes in the ISF. Overall, the internal gas motions within 

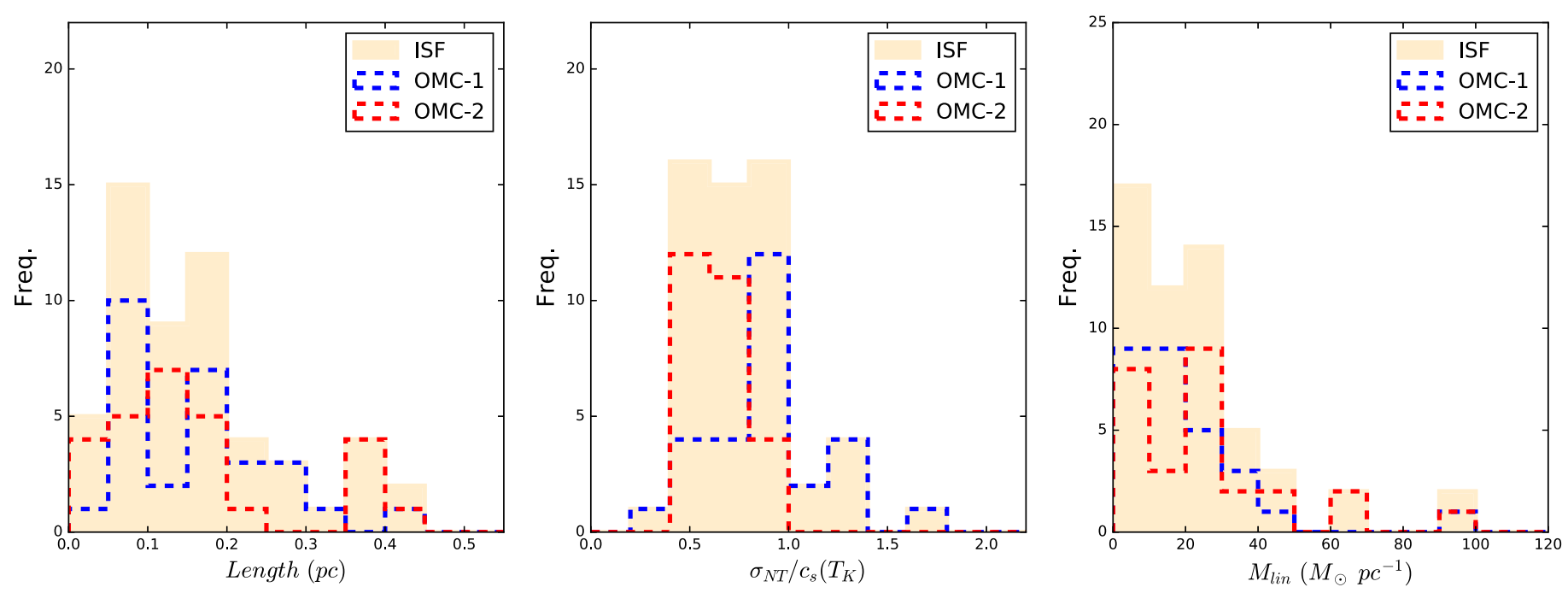

Fig. 5. Statistical properties of the ISF fibers (orange shaded area): left: total fiber length; centre: average line-of-sight, non-thermal velocity dispersion $\sigma_{N T}$ as a function of the local sound speed $\mathrm{c}_{s}\left(T_{\mathrm{K}}\right)$; and right: total mass per unit length. The individual fiber properties in OMC-1 and OMC-2 are highlighted by blue and red dashed lines, respectively.

Table 1. Dense gas properties.

\begin{tabular}{cccc}
\hline \hline & ISF & OMC-1 & OMC-2 \\
\hline $\mathrm{N}_{2} \mathrm{H}^{+}$fits $(S / N \geq 3)$ & 25078 & 13305 & 11773 \\
$T_{\mathrm{K}}(\mathrm{K})^{a}$ & $24.8 \pm 10.3$ & $29.0 \pm 12.5$ & $20.1 \pm 2.2$ \\
$\left\langle\mathrm{c}_{s}\left(T_{\mathrm{K}}\right)\right\rangle\left(\mathrm{km} \mathrm{s}^{-1}\right)$ & 0.294 & 0.318 & 0.267 \\
$\Delta V\left(\mathrm{~km} \mathrm{~s}^{-1}\right)$ & 0.607 & 0.722 & 0.477 \\
$\left\langle\sigma_{N T}\right\rangle\left(\mathrm{km} \mathrm{s}^{-1}\right)$ & 0.240 & 0.288 & 0.185 \\
$\left\langle\sigma_{N T} / \mathrm{c}_{s}\left(T_{\mathrm{K}}\right)\right\rangle$ & 0.81 & 0.91 & 0.69 \\
$\sigma_{N T} / \mathrm{c}_{s}\left(T_{\mathrm{K}}\right) \leq 1$ & $76.4 \%$ & $68.4 \%$ & $85.5 \%$ \\
$1<\sigma_{N T} / \mathrm{c}_{s}\left(T_{\mathrm{K}}\right) \leq 2$ & $20.0 \%$ & $25.7 \%$ & $13.6 \%$ \\
$\sigma_{N T} / \mathrm{c}_{s}\left(T_{\mathrm{K}}\right)>2$ & $3.6 \%$ & $5.9 \%$ & $0.9 \%$ \\
\hline
\end{tabular}

Notes. ${ }^{(a)}$ Ammonia-derived gas kinetic temperatures obtained from the GAS survey (Friesen et al. 2017).

this filament are described by an average non-thermal velocity dispersion of $\left\langle\sigma_{N T} / \mathrm{c}_{S}\left(T_{\mathrm{K}}\right)\right\rangle=0.81$. Remarkably, $>75 \%$ of the positions detected in $\mathrm{N}_{2} \mathrm{H}^{+}$show subsonic velocity dispersions (i.e., $\left.\sigma_{N T} / \mathrm{c}_{s}\left(T_{\mathrm{K}}\right) \leq 1\right)$. Conversely, less than $4 \%$ of the $\mathrm{N}_{2} \mathrm{H}^{+}$ components exhibit supersonic motions (i.e., $\sigma_{N T} / \mathrm{c}_{s}\left(T_{\mathrm{K}}\right)>2$ ). Our analysis therefore indicates that most of the dense gas within the massive ISF is relatively quiescent, typically showing subsonic $(>75 \%)$ or subsonic+transonic $(>95 \%)$ non-thermal motions.

In Fig. 5 (center), we show the histogram for the mean $\sigma_{N T}$ values (in units of the corresponding local $\mathrm{c}_{s}\left(T_{\mathrm{K}}\right.$ ); orange filled histogram) for all the fibers extracted by HiFIVE. The ISF fibers exhibit non-thermal velocity dispersions between 0.4 and 1.5 times their local sound speed. Low, sonic-like internal motions have been reported as an intrinsic characteristic of fibers in previous studies (Hacar \& Tafalla 2011; Hacar et al. 2013, 2017b). This unique property is also shared by the ISF fibers, where all the fibers detected in this massive filament are dominated by (tran-)sonic internal motions.

The small velocity dispersions measured in the ISF fibers appear to be in contradiction with the large linewidths reported in active regions like OMC-1 (e.g., Friesen et al. 2017). Our observations suggest that most of these broad emission lines detected in tracers like $\mathrm{N}_{2} \mathrm{H}^{+}$(Tatematsu et al. 2008) and $\mathrm{NH}_{3}$
(Friesen et al. 2017) are the result of a combination of multiple gas components and local velocity gradients typically unresolved within a single-dish beam. We illustrate this effect in Fig. 6 by comparing the emission in a single position recovered using different effective beam sizes. At the native ALMA resolution (red spectrum; upper panel), the observed $\mathrm{N}_{2} \mathrm{H}^{+}$emission shows three independent narrow lines, clearly separated in the hyperfine isolated component (gray dashed segments). Due to the complex gas kinematics within this region, the above line substructure is progressively smeared out when convolved with neighbouring positions to the resolution of previous single-dish studies (e.g., blue spectrum; central panel). Dilution and blending effects give the appearance of a single broad component at even larger beam sizes (black spectrum; lower panel). The above comparisons highlight the importance of both spectral and spatial resolution in the kinematic analysis of massive clouds.

\subsection{Linear masses and stability}

Characterizing the stability of the ISF fibers requires the study of their internal mass distribution. In the absence of additional line information, we have calibrated our $\mathrm{N}_{2} \mathrm{H}^{+}$emission with previous Herschel surveys along the ISF region. Introduced in similar studies using $\mathrm{N}_{2} \mathrm{H}^{+}$as line tracer (Tafalla \& Hacar 2015; Hacar et al. 2017b), this method obtains an empirical conversion factor between the observed integrated $\mathrm{N}_{2} \mathrm{H}^{+}$intensities and the equivalent gas plus dust column density. Detailed comparisons with radiative transfer Monte-Carlo simulations prove the validity of this technique in the case of optically thin emission (see also Tafalla \& Hacar 2015).

Figure 7 illustrates a point-to-point comparison of the total $\mathrm{N}\left(\mathrm{H}_{2}\right)$ column density derived in previous Herschel-Planck studies $\left(\theta_{F W H M}=36^{\prime \prime}\right.$; Lombardi et al. 2014) with similar single-dish $\mathrm{N}_{2} \mathrm{H}^{+}$integrated intensity maps $\left(\theta_{F W H M}=30^{\prime \prime}\right.$; Hacar et al. $2017 \mathrm{a}$ ), normalized by the corresponding gas kinetic temperature from the GAS-NH $\mathrm{NH}_{3}$ survey $\left(\theta_{F W H M}=32^{\prime \prime}\right.$; Friesen et al. 2017) (red filled circles) ${ }^{3}$. With the exception of several noisy positions in OMC-1 (see blue circles), the normalized $\mathrm{N}_{2} \mathrm{H}^{+}$intensities

3 We have excluded those positions within $R$ (Trapezium) $<0.3 \mathrm{pc}$ in Fig. 7 due to saturation effects in the Herschel-Planck maps (see Lombardi et al. 2014). 


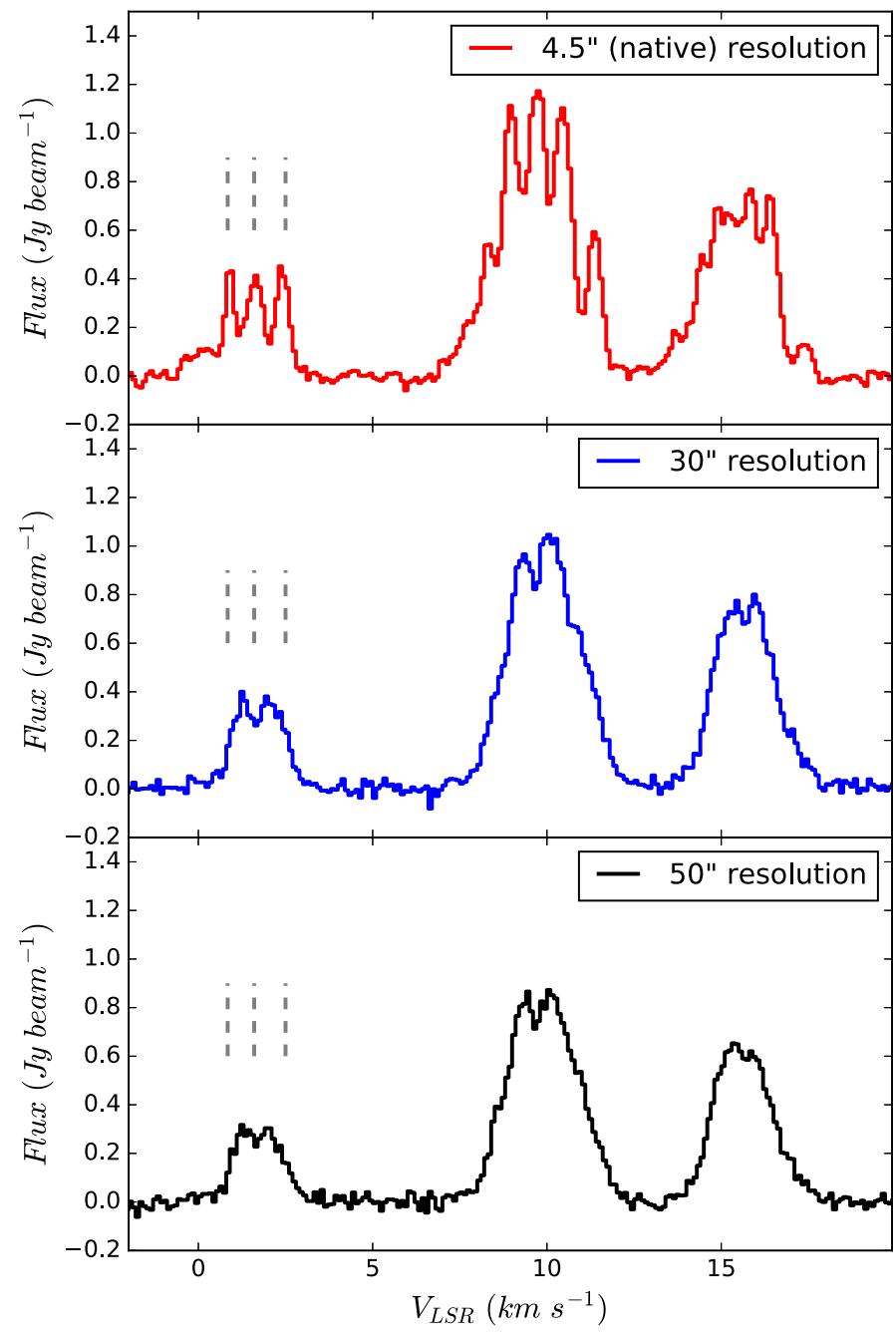

Fig. 6. Resolution effects affecting the identification of spectral line components at different resolutions: $\theta_{F W H M}=4.5^{\prime \prime}$ (native; upper panel), $\theta_{F W H M}=30^{\prime \prime}$ (mid panel), and $\theta_{F W H M}=50^{\prime \prime}$ (lower panel). For comparison, all spectra include the same noise level. We note how the three original velocity components, clearly detected in the $\mathrm{N}_{2} \mathrm{H}^{+}$ isolated hyperfine line at the native resolution (gray dashed lines), are progressively diluted and blended when convolved into larger beams. In particular, we note that most of the original line multiplicity is lost at the typical resolution of single-dish observations (mid panel; e.g. Hacar et al. 2017b).

exhibit a roughly linear correlation with the observed HerschelPlanck total column densities along the ISF. We have described this relationship using a least-squares fit of all the points included in Fig. 7 (black line) resulting in a linear term:

$\mathrm{N}\left(\mathrm{H}_{2}\right)\left[\mathrm{cm}^{-2}\right]=67.4 \times 10^{21} \cdot\left(\frac{I\left(\mathrm{~N}_{2} \mathrm{H}^{+}\right)\left[\mathrm{K} \mathrm{km} \mathrm{s}^{-1}\right]}{T_{\mathrm{K}}[\mathrm{K}]}\right)$.

Within a factor of two, this fit reproduces the observed correlation for column densities between $\mathrm{N}\left(\mathrm{H}_{2}\right) \sim(20-200) \times 10^{21} \mathrm{~cm}^{-2}$. Interestingly, we find an excellent correspondence between Eq. (2) in Orion and the results obtained from the study of the $\mathrm{N}_{2} \mathrm{H}^{+}$emission in the NGC 1333 region in Perseus (Hacar et al. 2017b) assuming a uniform $\mathrm{T}_{K}$ of $10 \mathrm{~K}$ (i.e., $\mathrm{N}\left(\mathrm{H}_{2}\right)=78.8 \times 10^{21} \cdot\left(\frac{I\left(\mathrm{~N}_{2} \mathrm{H}^{+}\right)}{10 \mathrm{~K}}\right)$; green dashed line). The good agreement between these two independent studies suggests similar abundances for $\mathrm{N}_{2} \mathrm{H}^{+}$ in both Orion and Perseus clouds and reinforces the use of

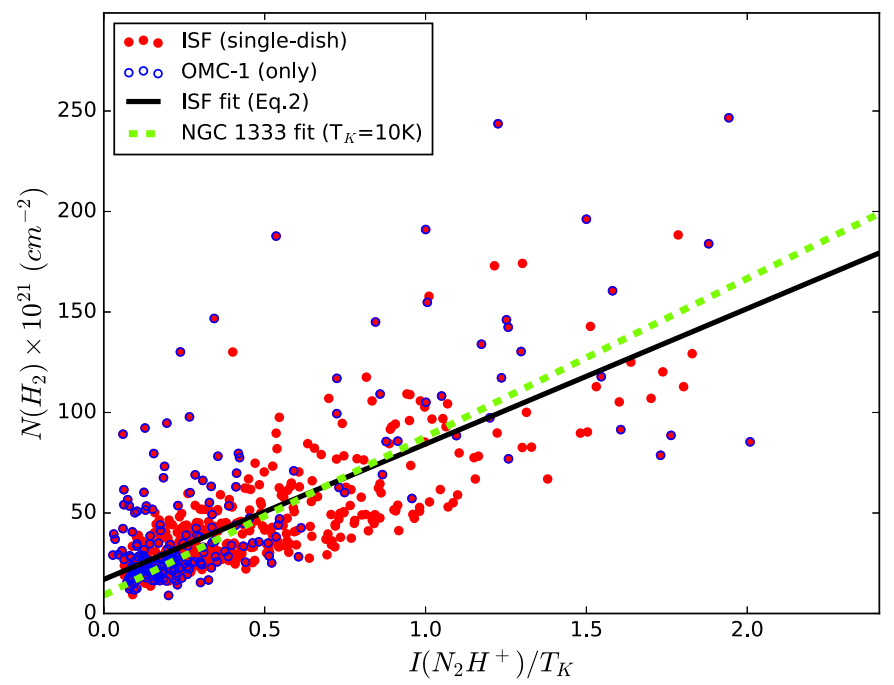

Fig. 7. Empirical correlation between the observed single-dish $\mathrm{N}_{2} \mathrm{H}^{+}$ intensities (Hacar et al. 2017a), normalized by the local gas kinetic temperature $T_{\mathrm{K}}$ (Friesen et al. 2017), and the total (gas + dust) column densities $\mathrm{N}\left(\mathrm{H}_{2}\right)$ derived in previous Herschel-Planck measurements (Lombardi et al. 2014). This plot includes all the positions detected at $R$ (Trapezium) $\geq 0.3 \mathrm{pc}$ in both OMC- 1 and OMC- 2 clouds (red dots). Those points belonging to the OMC-1 region are highlighted in blue. The thick black line indicates the results of the linear fit defining Eq. (2). The green dashed line indicates the expected correlation for the observed $\mathrm{N}_{2} \mathrm{H}^{+}$intensities in the NGC1333 proto-cluster assuming a constant temperature of $10 \mathrm{~K}$ (Hacar et al. 2017b).

this molecule as a robust tracer for dense gas under different physical conditions (e.g., Forbrich et al. 2014; Pety et al. 2016; Kauffmann et al. 2017).

We derived the total gas plus dust column density for each of the gas components detected in our ALMA maps from their corresponding integrated $\mathrm{N}_{2} \mathrm{H}^{+}$emission using the empirical correlation described in Eq. (2) (see also Appendix C). The total mass per fiber is then estimated from the addition of all components associated to each individual structure at the resolution of our maps assuming a distance of $D=414 \mathrm{pc}$. Finally, for each of these fibers we derive the mass per unit length $\mathbf{M}_{\text {lin }}$ dividing the above total mass by their corresponding length obtained in Sect. 3.1. Our length measurements do not consider projection effects making our $\mathbf{M}_{\text {lin }}$ values upper limits. In addition, uncertainties of a factor $\sim 2$ are expected for all the above estimates according to the dispersion observed in Fig. 7. The wide variations of the gas and dust properties seen in the vicinity of the ONC lead to larger uncertainties within the OMC-1 cloud.

Several caveats should be considered in respect to the interpretation of our mass per unit length values in Orion. First, we empirically obtained a unique intensity-to-mass conversion factor (Eq. (2)) from the comparison of single-dish and Herschel surveys. Extrapolated to the ALMA resolution, our mass conversion assumes that the same correlation applies at different scales and in different gas parcels of this cloud. Also, and by construction, its calibration is linked to the absolute values and error estimates of the Herschel dust column densities as well as the gas kinetic temperatures derived at single-dish resolutions. On the other hand, our mass calculations adopt an optically thin approximation for $\mathrm{N}_{2} \mathrm{H}^{+}$emission in the ISF. Although justified by our opacity estimates in most cases, this assumption can selectively affect the individual masses of several fibers in our sample (see Appendices B.3 and C for a discussion). Some of 

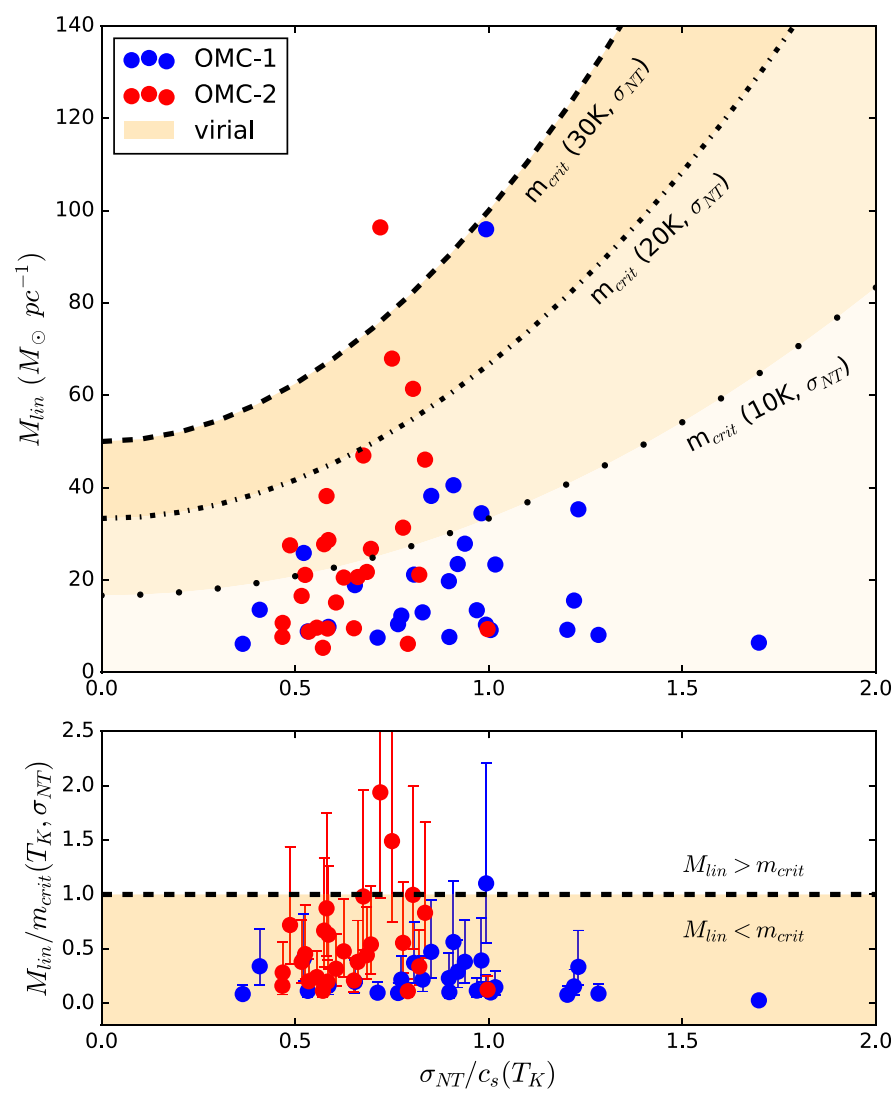

Fig. 8. Upper: mass per unit length $\left(\mathrm{M}_{\text {lin }}\right)$ of the OMC-1 (blue dots) and OMC-2 (red dots) fibers as function of their internal non-thermal velocity dispersion normalized by the local sound speed $\left(\sigma_{N T} / \mathrm{c}_{s}\left(T_{\mathrm{K}}\right)\right)$. This plot includes the expected critical masses $\left(m_{\text {crit }}\right)$ for an infinite filament in hydrostatic equilibrium at temperatures of $10 \mathrm{~K}$ (thick dashed line), $20 \mathrm{~K}$ (dashed-dotted line), and $30 \mathrm{~K}$ (thick dotted line), respectively (see Eq. (3)). Lower: individual critical mass ratio $\left(\mathrm{M}_{\text {lin }} / m_{\text {crit }}\right)$ for all the fibers in OMC-1 (blue) and OMC-2 (red). For every single fiber the critical mass ratio is calculated relative to its local $m_{\text {crit }}\left(T_{K}, \sigma_{N T}\right)$. The error bars indicate the factor of two uncertainties associated to our $\mathrm{M}_{\text {lin }}$ estimates.

the above assumptions are partially responsible for the observed spread in Fig. 7 and are assumed to be included in the factor 2-3 uncertainties estimated for the slope of Eq. (2). Despite our efforts, larger uncertainties cannot be ruled out, particularly in the OMC-1 fibers. Additional ALMA observations, including multiple transitions and tracers, are needed to better constrain these mass estimates.

As shown in Fig. 5 (right), the derived $\mathbf{M}_{\text {lin }}$ values for the ISF fibers typically range between 10 and $60 M_{\odot} \mathrm{pc}^{-1}$ with a median value of $\sim 19 M_{\odot} \mathrm{pc}^{-1}$. Three exceptions are found showing $\mathrm{M}_{\text {lin }}>60 M_{\odot} \mathrm{pc}^{-1}$, namely, fibers \# 21 (OMC-1 ridge; $\left.\mathrm{M}_{\text {lin }}=95 M_{\odot} \mathrm{pc}^{-1}\right)$, \# $37\left(\mathrm{OMC}-2\right.$ FIR-6; $\left.\mathrm{M}_{\text {lin }}=96 M_{\odot} \mathrm{pc}^{-1}\right)$, and \# 42 (OMC-2 FIR-4; $\left.\mathrm{M}_{\text {lin }}=68 M_{\odot} \mathrm{pc}^{-1}\right)$. As discussed in Appendix A, these three regions present clear signs of a complex substructure not recovered by our HiFIVE algorithm. Their unusually large mass per unit length should be then taken as upper limits of their actual mass properties.

In Fig. 8 (upper panel), we represent the dynamical state of the ISF fibers in comparison to their expected masses in equilibrium. For each individual fiber, we display the observed $\mathbf{M}_{l i n}$ as a function of the normalized non-thermal velocity dispersion $\sigma_{N T} / \mathrm{c}_{s}\left(T_{\mathrm{K}}\right)$ (see Sect. 3.2). We also represent the expected critical mass $\left(m_{\text {crit }}\right)$ for an infinite filament in hydrostatic equilibrium
(Stodólkiewicz 1963; Ostriker 1964):

$m_{c r i t}\left(T_{\mathrm{K}}, \sigma_{N T}\right)=\frac{2 \sigma_{e f f}^{2}}{G}=\frac{2 \mathrm{c}_{s}\left(T_{\mathrm{K}}\right)^{2}}{G}\left(1+\left(\frac{\sigma_{N T}}{\mathrm{c}_{s}\left(T_{\mathrm{K}}\right)}\right)^{2}\right)$,

where $\sigma_{e f f}^{2}=\mathrm{c}_{s}\left(T_{\mathrm{K}}\right)^{2}+\sigma_{N T}^{2}$ is defined as the effective (thermal + non-thermal) velocity dispersion. Typically, $m_{\text {crit }}$ is assumed to correspond with the expected value for a thermally supported filament at $T_{\mathrm{K}}=10 \mathrm{~K}, m_{\text {crit }}\left(\sigma_{\text {eff }}=\mathrm{c}_{s}(10 \mathrm{~K})\right)=16.6 M_{\odot} \mathrm{pc}^{-1}$. However, this simplified analysis neglects the additional support provided by non-thermal motions and systematically higher temperatures found in active star-forming regions like Orion. To properly evaluate these effects, in Fig. 8 (upper panel) we superpose the evolution of the expected critical masses for filaments at temperatures of $10 \mathrm{~K}$ (dotted line), $20 \mathrm{~K}$ (dot-dashed line), and $30 \mathrm{~K}$ (dashed line) including the additional $\sigma_{N T}$ contributions in Eq. (3). As denoted in this plot, the vast majority of the ISF fibers have subcritical masses (i.e., $\mathrm{M}_{\text {lin }} \lesssim m_{\text {crit }}\left(T_{\mathrm{K}}, \sigma_{N T}\right)$; shaded areas) for temperatures between 20 and $30 \mathrm{~K}$, similar to those reported in OMC-1 and OMC-2 (see Table 1).

To facilitate their comparison, in Fig. 8 (lower panel) we display the individual ratios of the observed $\mathbf{M}_{\text {lin }}$ values with respect to their local critical mass $m_{c r i t}\left(T_{\mathrm{K}}, \sigma_{N T}\right)$ in all the ISF fibers. Each measurement includes a factor of two variation on the $\mathrm{M}_{\text {lin }}$ estimates (error bars; see above). In $\sim 50 \%$ of the cases, the ISF fibers show (within the errors) critical-mass ratios between $0.5 \leq \mathrm{M}_{\text {lin }} / m_{\text {crit }} \leq 1.5$. Our results suggest that these fibers are gravitationally bound and largely supported by a combination of thermal and (sonic) turbulent motions presenting a configuration consistent with (or close to) equilibrium. This quasi-stable radial configuration in fibers would provide the necessary conditions for their subsequent fragmentation into cores (Inutsuka \& Miyama 1997; Heigl et al. 2016). Possible exceptions to this average behavior are found in the most massive fibers \# 21, 37, and 42, showing masses potentially exceeding $\mathrm{M}_{\text {lin }} / m_{\text {crit }} \gtrsim 1.5$. While still unresolved in our observations, these high mass ratios suggest that they are gravitationally unstable. In contrast, up to $\sim 50 \%$ of fibers show $\mathrm{M}_{\text {lin }} / m_{\text {crit }} \leq 0.5$ and are, therefore, gravitationally unbound (e.g., see some of the OMC-1 fibers). In the absence of additional confinement (i.e. thermal pressure, accretion, and/or tidal forces) these latter fibers may be transient objects.

\subsection{Fiber widths: typical $0.03 p c$ values}

The high dynamic range of our $\mathrm{N}_{2} \mathrm{H}^{+}$ALMA mosaics reveals a unique characteristic of the ISF fibers. As clearly seen in the zoom-in maps of Fig. 3, most fibers exhibit a compact radial profile showing a sharp emission contrast compared to their local background. Several examples of this behavior can be found in the OMC-1 Ridge, the OMC-1 South, and the OMC-2 FIR-6 regions. Remarkably, all fibers show extremely narrow widths at scales $\ll 0.1$ pc with a sharp emission drop of equivalent column densities $>50 \mathrm{~A}_{V}$ on scales comparable to our beamsize (i.e., $0.009 \mathrm{pc}$; see some of the molecular fingers along the OMC-1 Ridge). We remind the reader here that the combination of ALMA plus (zero-spacing) single-dish data minimizes potential biases produced by spatial filtering effects (Sect. 2). In combination with the optically thin emission properties of $\mathrm{N}_{2} \mathrm{H}^{+}$(Appendix B.3), the above results describe the intrinsically compact mass distribution within fibers.

In Fig. 9, we illustrate the ISF fiber widths from the detailed study of several of these objects in both OMC-2 South (upper panel) and OMC-1 Ridge (lower panel) subregions. These fibers 

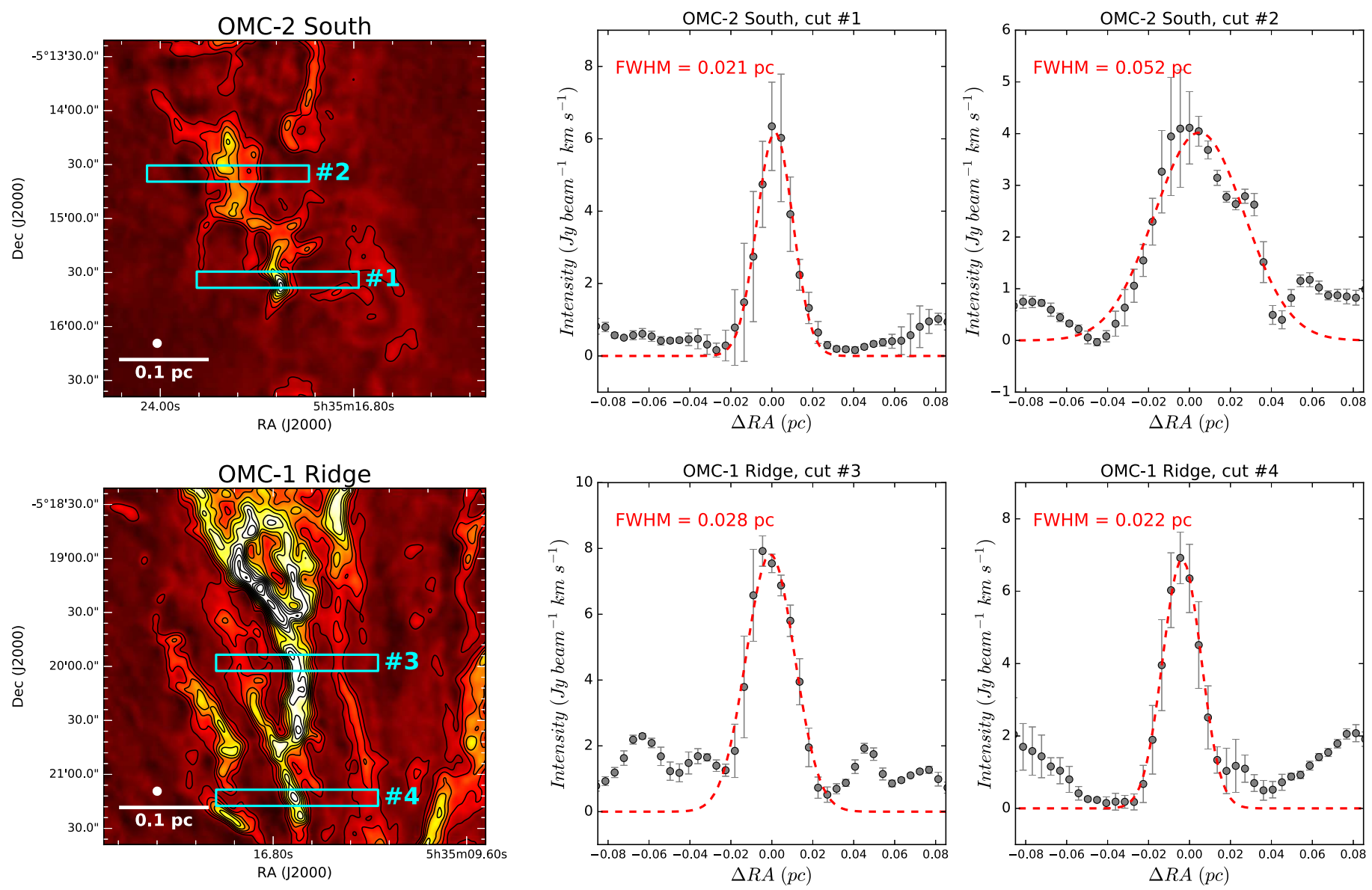

Fig. 9. Typical fiber widths in both OMC-2 South (upper panel; fibers \#30 and 34) and OMC-1 Ridge (lower panel; fibers \#19 \& \#21) regions. Left subpanels: total integrated $\mathrm{N}_{2} \mathrm{H}^{+}$emission including the selected cuts (\# 1-4) perpendicular to the fibers (cyan boxes). Contours are equally spaced every $1 \mathrm{Jy}_{\text {beam }}^{-1} \mathrm{~km} \mathrm{~s}^{-1}$. Center and right subpanels: average $\mathrm{N}_{2} \mathrm{H}^{+}$emission along cuts \# $1-4$ (gray dots with errors). The red dashed line indicates the Gaussian fits for each profile (see FWHM in the top-left corner of each subplot).

were selected for their favorable geometry and orientation, with a clean and well-defined axis. In each case, we have extracted two horizontal cuts roughly perpendicular to the main fiber axis (cyan boxes in left panels). For the selected cuts $1-4$, in Fig. 9 (lateral subpanels) we show the corresponding total $\mathrm{N}_{2} \mathrm{H}^{+}$ integrated emission as a function of Right Ascension (RA) centered at the position of the corresponding fiber axis. We estimate the typical fiber widths within these regions by fitting a single Gaussian function to each of the above cuts (red dashed lines). As seen in the different subpanels, most of the observed fiber radial profiles can be well described by a Gaussian distribution with a full-width-at-half-maximum FWHM between 0.02 and $0.03 \mathrm{pc}$ (cuts 1, 3, and 4). Asymmetric and complex profiles complicate this analysis producing a broader FWHM of $\sim 0.05 \mathrm{pc}$ (see cut 3).

Similar to the above examples, we have statistically characterized the observed fiber widths using regular cuts perpendicular to the main fiber axes defined in Fig. 4. We carried out this analysis in our total $\mathrm{N}_{2} \mathrm{H}^{+}$integrated intensity maps (Fig. 2, right panel). Although potentially contaminated by fiber superpositions in some localized positions, this approach is preferred because of the better stability of these integrated maps compared to the slightly noisier measurements derived from the line fits as well as for the inclusion of the full intensity emission profile and not only high-S/N data. Figure 10 (left) shows the median (solid red line) and total range (min-max; red shaded area) of the observed FWHM along all the ISF fibers (576 cuts). At each position, the reported FWHM values are obtained from the Gaussian fit of the total integrated $\mathrm{N}_{2} \mathrm{H}^{+}$emission within the innermost $0.05 \mathrm{pc}$ region around the fiber axis. The ISF fibers show a median FWHM of 0.035 pc (blue dashed line) with $85 \%$ of the cuts showing FWHM values $<0.050 \mathrm{pc}$ (see histogram in Fig. 10 right). In contrast, less than $1 \%$ of these cuts exhibit $F W H M>0.1 \mathrm{pc}$. Systematic width variations of a factor $\sim 2-3$ are observed both between and within fibers. Larger widths are found in complex regions (e.g., fiber \# 23) or in structures with ill-defined axes (e.g., fiber \# 33). At the opposite end, several fibers are marginally resolved at the resolution of our ALMA observations, showing FWHM values of $\sim 0.02$ pc (e.g., fibers \# 15 and 24).

Recent Herschel observations in nearby clouds like IC5146, Polaris, or Taurus have suggested the existence of a constant filament width of $\sim 0.1$ pc (Arzoumanian et al. 2011; André et al. 2014; Palmeirim et al. 2013). Independent studies have pointed out several observational biases affecting these measurements, questioning the robustness of these results (Smith et al. 2014; Panopoulou et al. 2017). Interferometric studies in clustered star-forming regions have also reported the detection of several elongated (fiber-like) substructures showing FWHMs below $<0.05$ pc (Pineda et al. 2011; Fernández-López et al. 2014; Henshaw et al. 2017). The observed properties of the ISF fibers add new and direct evidence of a systematic departure from this 

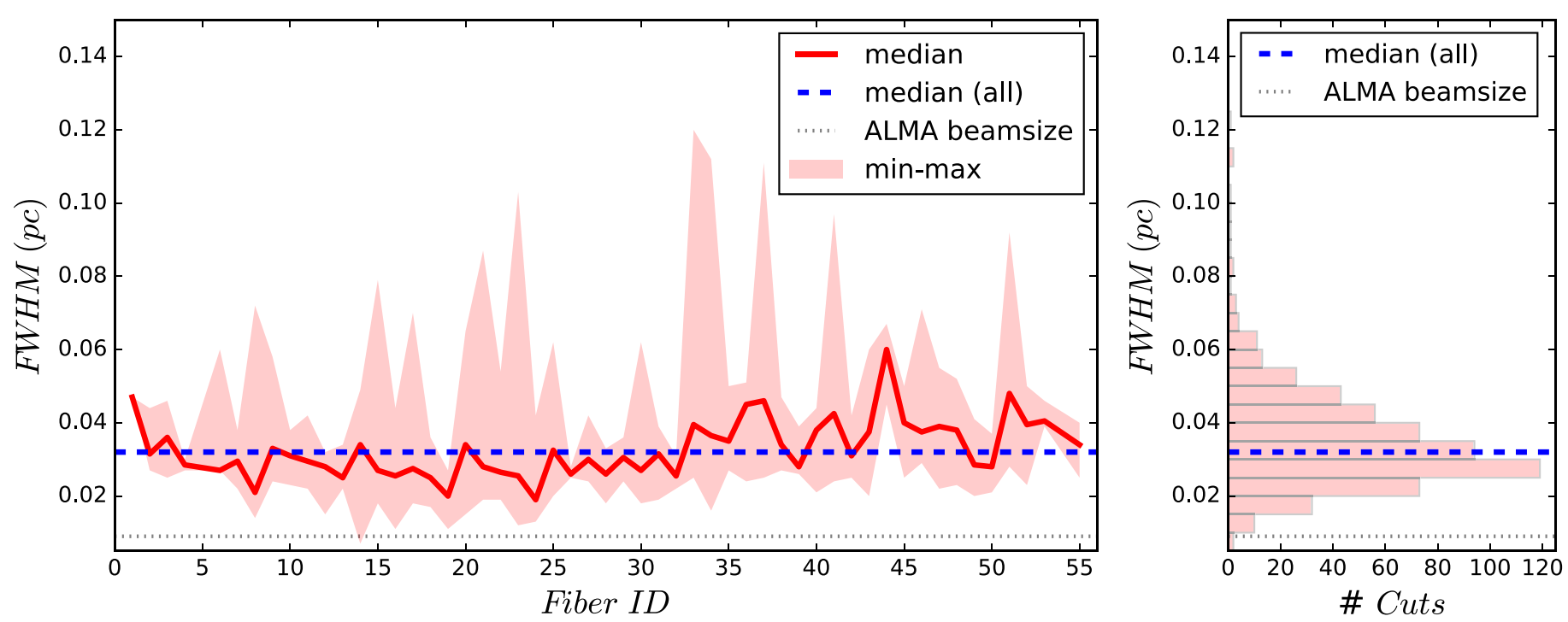

Fig. 10. Observed fiber FWHM along the ISF. Left: median (red solid line) and minimum-maximum (red shaded area) FWHM values obtained in each fiber. Right: total FWHM values (576 data points; red histogram) measured along the ISF fibers. The total median value of $0.035 \mathrm{pc}$ (blue dashed line) in comparison with the ALMA 0.009 pc beamsize (grey dotted line) are indicated in both panels.

Table 2. Fiber properties in molecular clouds.

\begin{tabular}{|c|c|c|c|c|c|c|}
\hline & $\mathrm{ISF}^{1}$ & OMC $-1^{1}$ & OMC- $2^{1}$ & $\mathrm{NGC} 1333^{2}$ & B213-L1495 ${ }^{3, \star}$ & Musca $^{4, \#}$ \\
\hline Region & Orion & Orion & Orion & Perseus & Taurus & Musca \\
\hline Distance (pc) & 414 & 414 & 414 & 236 & 140 & 140 \\
\hline Mass range & High-mass & High-mass & Intermediate & Intermediate/Low & Low & Low \\
\hline Mode & Clustered & Clustered & Clustered & Clustered & Isolated & Isolated \\
\hline $\mathrm{M}_{\text {lin }}($ total $)\left(M_{\odot} \mathrm{pc}^{-1}\right)$ & $\sim 500^{(6,7)}$ & $\sim 500^{(6,7)}$ & $\sim 500^{(6,7)}$ & $\sim 200$ & $\sim 50^{(8)}$ & $\sim 25^{(5)}$ \\
\hline Observations & ALMA & ALMA & ALMA & IRAM 30m & FCRAO & APEX \\
\hline$\theta_{F W H M}\left({ }^{\prime \prime}\right)$ & 4.5 & 4.5 & 4.5 & 30 & 60 & 28.5 \\
\hline Resolution (pc) & 0.009 & 0.009 & 0.009 & 0.034 & 0.040 & 0.019 \\
\hline \# Fertile fibers & 55 & 28 & 27 & 14 & 7 & 1 \\
\hline Size $(\mathrm{pc})$ & $2.5 \times 0.48$ & $1.0 \times 0.48$ & $1.5 \times 0.48$ & $2.0 \times 0.4$ & $10.0 \times 1.0$ & $6.5 \times 0.5$ \\
\hline Area $\left(\mathrm{pc}^{2}\right)$ & 1.2 & 0.48 & 0.72 & 0.8 & 10 & 3.25 \\
\hline$\Sigma($ fertile $)\left(\mathrm{pc}^{-2}\right)$ & 45.8 & 58.3 & 37.5 & 17.5 & 0.7 & 0.3 \\
\hline FWHM (pc) & $\sim 0.02-0.05$ & $\sim 0.02-0.05$ & $\sim 0.02-0.05$ & $\sim 0.1$ & $\sim 0.1^{(8)}$ & $0.07^{(5)}$ \\
\hline$\langle$ Length $\rangle(\mathrm{pc})$ & $0.16 \pm 0.10$ & $0.15 \pm 0.09$ & $0.16 \pm 0.11$ & $0.4 \pm 0.2$ & $1.0 \pm 0.2$ & 6.5 \\
\hline$\left\langle\mathrm{M}_{\text {lin }}\right\rangle\left(M_{\odot} \mathrm{pc}^{-1}\right)$ & $23 \pm 11$ & $20 \pm 18$ & $26 \pm 21$ & $34 \pm 22$ & $24 \pm 19$ & 26 \\
\hline$\left\langle n\left(\mathrm{H}_{2}\right)\right\rangle\left(\mathrm{cm}^{-3}\right)$ & $1.0 \times 10^{8}$ & $0.8 \times 10^{8}$ & $1.2 \times 10^{8}$ & $\sim 5 \times 10^{5}$ & $\sim 10^{5}$ & $\sim 10^{4}$ \\
\hline$\left\langle T_{\mathrm{K}}\right\rangle(\mathrm{K})$ & $24.7 \pm 6.7$ & $28.9 \pm 7.1$ & $20.4 \pm 1.5$ & $\sim 10$ & $\sim 10$ & $\sim 10$ \\
\hline$\left\langle\sigma_{N T} / c_{s}\left(T_{\mathrm{K}}\right)\right\rangle$ & $0.77 \pm 0.25$ & $0.89 \pm 0.28$ & $0.65 \pm 0.13$ & $1.0 \pm 0.3$ & $1.1 \pm 0.3$ & 0.7 \\
\hline$\left\langle\left.\nabla V_{L S R}\right|_{\text {global }}\right\rangle\left(\mathrm{km} \mathrm{s}^{-1} \mathrm{pc}^{-1}\right)$ & $4.6 \pm 5.9$ & $6.5 \pm 7.4$ & $2.6 \pm 2.3$ & $0.8 \pm 0.8$ & $0.4 \pm 0.4$ & 0.3 \\
\hline$\left\langle\left.\nabla V_{L S R}\right|_{\text {local }}\right\rangle\left(\mathrm{km} \mathrm{s}^{-1} \mathrm{pc}^{-1}\right)$ & $12.8 \pm 7.7$ & $16.1 \pm 9.2$ & $9.5 \pm 3.3$ & $1.5 \pm 0.6$ & $1.3 \pm 0.5$ & $\sim 1-2$ \\
\hline
\end{tabular}

Notes. The values listed in this table correspond to the mean and 1- $\sigma$ dispersion in each case. We notice that not all the above parameters can be described by a normal distributions so the use of a gaussian dispersion is only indicative of the observed varability.

References: ${ }^{(1)}$ This work; ${ }^{(2)} \mathrm{Hacar}$ et al. (2017b); ${ }^{(3)} \mathrm{Hacar}$ et al. (2013); ${ }^{(4)} \mathrm{Hacar}$ et al. $(2016) ;{ }^{(5)}$ Kainulainen et al. (2016); ${ }^{(6)} \mathrm{Bally}$ et al. (1987);

(7) Johnstone \& Bally (1999); ${ }^{(8)}$ Palmeirim et al. (2013); ${ }^{(\star)}$ Only fertile fibers detected in $\mathrm{N}_{2} \mathrm{H}^{+}$are considered. ${ }^{(\#)}$ Assumed as single fertile fiber (see Hacar et al. 2016, for a discussion).

"universal" behavior. With widths ranging between $\sim 0.02$ and $0.05 \mathrm{pc}$, our statistical results undoubtedly prove the existence of filamentary structures with radial FWHM significantly narrower than the previously proposed $0.1 \mathrm{pc}$ scale.

The compact widths of the ISF fibers could be related to the higher densities found in Orion when compared to other low-mass star-forming regions explored by Herschel. Although highly idealized, it is instructive to compare the expected variations of the scale height of a filament in hydrostatic equilibrium $\left(H_{0}\right)$ as function of the gas temperature $\left(T_{\mathrm{K}}\right)$, nonthermal motions $\left(\sigma_{N T}\right)$, and density $\left(n\left(\mathrm{H}_{2}\right)\right)$ (Ostriker 1964):

$H_{0}\left(T_{\mathrm{K}}, n\left(\mathrm{H}_{2}\right)\right)=\sqrt{\frac{2 \sigma_{e f f}^{2}}{\pi G n\left(\mathrm{H}_{2}\right)}} \propto \sqrt{\frac{\mathrm{c}_{s}\left(T_{\mathrm{K}}\right)^{2}+\sigma_{N T}^{2}}{n\left(\mathrm{H}_{2}\right)}}$.

Following this equilibrium solution, denser filaments are thus expected to show narrower radial configurations. For the 
ISF fibers, and consistent with our line opacity measurements (see Appendix B.3), we find typical gas densities of $n\left(\mathrm{H}_{2}\right) \sim 10^{7}-10^{8} \mathrm{~cm}^{-3}$ assuming a cylindrical symmetry and the previously derived $\mathrm{M}_{\text {lin }}$ and FWHM values (see Table 2 ). These estimates are (at least) 2 orders of magnitude higher than the densities measured for typical low-mass filaments in clouds like IC5146, Polaris, or Taurus. According to Eq. (4), the observed radii in both the ISF fibers $\left(T_{\mathrm{K}} \sim 25 \mathrm{~K} ; \sigma_{N T}=\mathrm{c}_{s}(25 \mathrm{~K})\right.$; $n\left(\mathrm{H}_{2}\right)=10^{8} \mathrm{~cm}^{-3}$ ) and Herschel filaments (e.g., Herschel: $\left.T_{\mathrm{K}}=10 \mathrm{~K} ; \sigma_{N T}=\mathrm{c}_{s}(10 \mathrm{~K}) ; n\left(\mathrm{H}_{2}\right)=5 \times 10^{5} \mathrm{~cm}^{-3}\right)$ are expected to show a size dependency such that $\frac{H_{0}(\mathrm{Herschel})}{H_{0}(\mathrm{ISF})} \sim 9$. Although deviating from these simplified predictions, the observed width ratio $\frac{F W H M(\text { Herschel })}{F W H M(\text { ISF })}=\frac{0.1}{\sim 0.03} \sim 3-4$ indicates that fibers might present a wide range of intrinsic widths depending on the initial gas densities.

\subsection{Environmental effects: OMC-1 versus OMC-2}

So far, we have considered the entire ISF as a single star-forming region based on its continuity at large scales (e.g., Bally et al. 1987). In addition to this global analysis, the wide-field coverage of our ALMA mosaics allows us to investigate the potential impact of distinct feedback and dynamical effects on the ISF fibers. Indeed, both thermal and kinematic gas properties in the OMC-1 cloud are directly influenced by the ONC activity and its global gravitational collapse (Hacar et al. 2017b) compared to the more pristine conditions expected for the OMC-2 region. In this Section, we explore these environmental effects by studying the fiber properties in each OMC-1 and OMC- 2 clouds independently.

In Fig. 5, we display the distribution of the fiber lengths (left), average 1.o.s. velocity dispersions (center), and mass per unit lengths (right) in both OMC-1 (blue) and OMC-2 regions (red). We also list the mean and 1- $\sigma$ dispersion values in Table 2 (see Cols. 2 and 3). Overall, we find no systematic differences between these properties along the ISF. On average, OMC-1 and OMC-2 regions have fibers with statistically similar lengths, velocity dispersion, and mass per unit length. Minor variations are, however, apparent in Fig. 5 (left) and (center). The OMC-1 fibers appear to show larger velocity dispersions and gradients than those in OMC-2 (see additional parameters in Table 2). While appealing, these differences are found within the 1- $\sigma$ dispersion estimates and can only be considered as tentative. Despite these local variations, the observed ISF fibers are found to present roughly uniform properties regardless of their local environment.

Morphologically speaking, the ISF fibers are distributed in multiple hub-like associations (Myers 2009) in both OMC-1 and OMC-2 regions. As seen in Fig. 4, most OMC-1 fibers are oriented radially converging towards the OMC-1 South region (see Rodriguez-Franco et al. 1992; Wiseman \& Ho 1998). Although partially recovered in our fiber analysis, similar fan-like arrangements of fibers can also be recognized at smaller scales towards the centers of OMC-2 FIR 4, OMC-2 FIR 6, and OMC-1 Ridge (Fig. 3). These local properties are highlighted in comparison with the sparse distribution of fibers outside these regions (e.g., along OMC-2 FIR 1). The above fiber arrangements seem to be created by gravitational focusing effects and to respond the local variations of the cloud potential (Hartmann \& Burkert 2007; Vázquez-Semadeni et al. 2017; Kuznetsova et al. 2017). We note that all of the above fiber hubs coincide with the positions of different (stars + gas) mass concentrations along the ISF. Moreover, we find an apparent correspondence between the depth of the potential and the number of objects in these fiber arrangements, with more massive hubs fed by an increasing number of fibers (e.g., see OMC-1 in comparison with OMC-2 FIR 4). In several cases, we also observe an increase of the gas motions along the fibers in the proximity of these hubs as expected in a gravitationally dominated gas velocity field. In the most prominent example, our high-resolution observations confirm the correlation between the orientation and global velocity gradients of the OMC-1 fibers induced by their global infall towards the current center of mass of this cloud located in the vicinity of the OMC-1 South proto-cluster (see green cross in Fig. 4; Hacar et al. 2017a, see also Appendix B.2). While additional kinematic analysis are needed to confirm these results, gravity appears to be the driving mechanism in shaping the spatial distribution of fibers within this cloud.

This fiber organization is reminiscent of the competitive accretion scenario for the formation of high-mass stars (Bonnell et al. 2007, and references therein). Rather than stellar objects, however, the location of the potential well would be selfgenerated by initial concentration of fibers. In this sense, multiple centers of collapse can be created depending on the complexity of these fiber networks (e.g., OMC-1 ridge, OMC-2 FIR 6 , or OMC-2 FIR) and evolve in time responding to changes in the local center of mass (e.g., from the ONC to OMC-1 South; see Hacar et al. 2017a).

\section{Discussion}

\subsection{Fiber properties in low- and high-mass filaments}

In combination with previous surveys, we systematically explore the variation of the fiber properties in both low- and high-mass filaments. We statistically compare these properties within a series of filamentary clouds with increasing total mass per unit length: (a) the isolated Musca filament (Hacar et al. 2016), (b) the low-mass L1495-B213 cloud in Taurus (Hacar et al. 2013), (c) the NGC 1333 ridge in Perseus (Hacar et al. 2017b), and both (d) the OMC-2 plus (e) the OMC-1 regions in Orion (this work). In Table 2 we summarize the main characteristics of the starforming fibers identified in these clouds. Typically detected in dense tracers like $\mathrm{N}_{2} \mathrm{H}^{+}$, those fibers forming cores and stars are classified as fertile (see Hacar et al. 2013, for a discussion) ${ }^{4}$. In Fig. 11 we display the corresponding cumulative distributions for the observed 1.o.s. non-thermal velocity dispersions (left), mass per unit length (centre), and fiber lengths (right) in all Musca (green dashed line), B213-L1495 (black dotted line), NGC 1333 (orange dashed line), OMC-2 (solid red line), and OMC-1 (solid blue line).

Across all regions, fibers are characterized by a low level of turbulence relative to their local thermal motions. As seen in Fig. 11 (left), fibers exhibit 1.o.s. non-thermal velocity dispersions ranging between $\sim 0.5$ and $\sim 1.5$ times their corresponding $\mathrm{c}_{s}\left(T_{\mathrm{K}}\right)$. We find no significant difference between fibers in clouds like Taurus, Perseus, and Orion, all showing similar average

\footnotetext{
4 Our selection criteria assume as fertile all fibers with emission in the density selective $\mathrm{N}_{2} \mathrm{H}^{+}$line. That includes all the fibers identified in both the ISF (55) and NGC 1333 (14) regions, but only 7 out of the 35 fibers identified in B213-L1495 (see Hacar et al. 2013). The other 28 fibers in B213-L1495, only detected at the lower densities traced by the $\mathrm{C}^{18} \mathrm{O}(1-0)$ line, are considered sterile and are not included in our calculations (see Table 2). Although no $\mathrm{N}_{2} \mathrm{H}^{+}$maps are available for Musca, we identify this filament as a single fertile fiber according to its fragmentation state showing multiple embedded prestellar cores and one protostar (Kainulainen et al. 2016).
} 

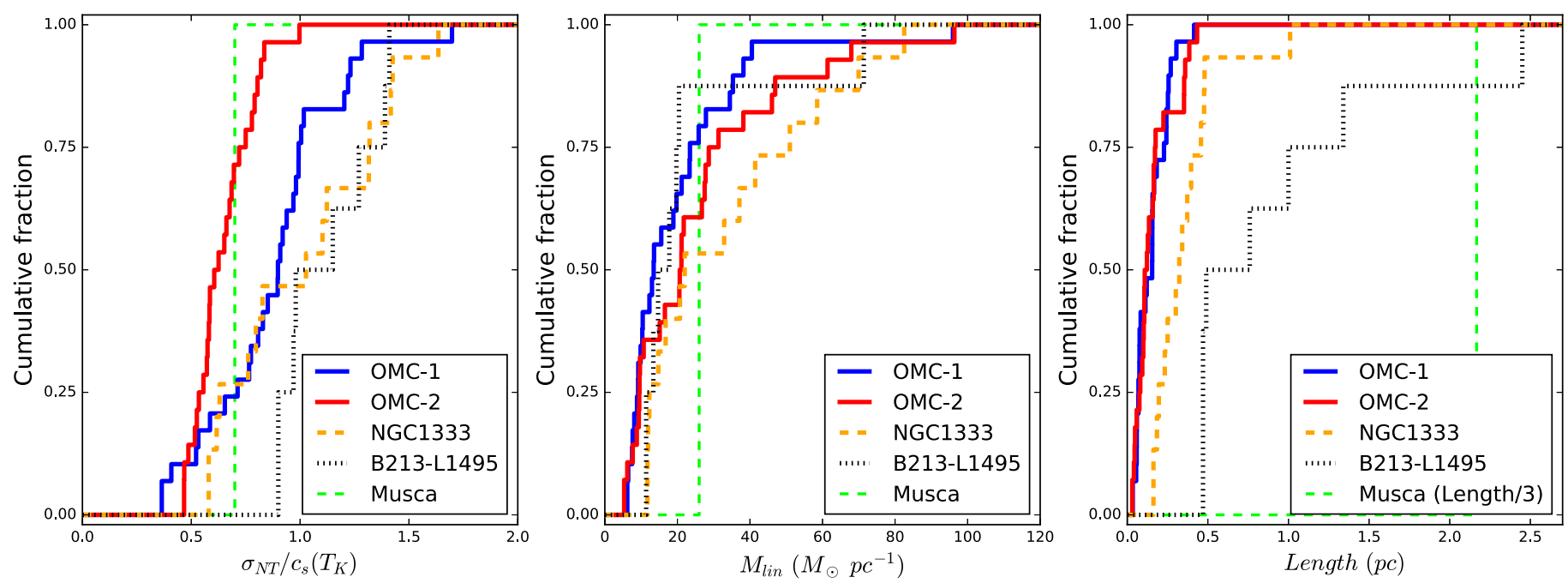

Fig. 11. Statistical properties of the fertile fibers in Musca (green dashed line), B213-L1495 (black dotted line), NGC1333 (orange dashed line), OMC-2 (red solid line), and OMC-1 (blue solid line) (see also Table 2). Cumulative distributions of the line-of-sight, non-thermal velocity dispersion $\sigma_{N T}$ as a function of the local sound speed $\mathrm{c}_{s}\left(T_{\mathrm{K}}\right)$ (left), mass per unit length (center), and total fiber lengths (right). We notice that the total length of Musca has been reduced by a factor of one third in the right panel.

$\left\langle\sigma_{N T} / \mathrm{c}_{s}\left(T_{\mathrm{K}}\right)\right\rangle$ values within the $1-\sigma$ error (see Table 2$)$. Their quiescent internal motions contrast with the broad linewidths commonly reported in these clouds. Our analysis indicates that most of these observational differences are created by a combination of global thermal effects, small-scale velocity gradients, and superposition, typically convolved in these previous singledish surveys (see Sect. 3.2). Once properly resolved, fibers show typical transonic motions (i.e., $1<\sigma_{N T} / \mathrm{c}_{s}\left(T_{\mathrm{K}}\right) \leq 2$ ) in both lowand high-mass clouds.

We also find a relatively good agreement between the distributions of the masses per-unit-length for fibers across the entire cloud spectrum. Most of the fibers in Fig. 11 (center) show consistent $\mathrm{M}_{\text {lin }}$ values between 10 and $\sim 80 M_{\odot} \mathrm{pc}^{-1}$ in the four clouds used for this comparison. Small deviations can be attributed to low-number statistics (e.g., Musca or B213-L1495) and the limitations of our analysis (e.g., OMC-1 Ridge; see Appendix A). In most cases, the reported $\mathrm{M}_{\text {lin }}$ values are consistent, within a factor of two, with the predicted masses for hydrostatic filaments internally supported by a combination of thermal and (sonic-like) turbulent motions (see Fig. 8). This apparent stable configuration might then explain the widespread detection of fibers over the wide range of conditions covered in these studies.

Contrary to the above similarities, we do find an environmental dependence on several of the observed fiber properties. First, fibers in massive regions show larger internal velocity gradients than those in low-mass clouds. This increase is evidenced in the average local velocity gradients $\left\langle\nabla V_{\mathrm{LSR} \mid \text { local }}\right\rangle$ found in OMC-1 and OMC-2, typically between three and five times higher than those measured in Musca or Taurus (see Table 2). Part of these variations can be potentially affected by resolution effects and the increase of local motions at small scales (e.g. Hacar \& Tafalla 2011). Nevertheless, the largest $\left\langle\nabla V_{\mathrm{LSR} \mid \text { local }}\right\rangle$ and $\left\langle\nabla V_{\mathrm{LSR} \mid \text { global }}\right\rangle$ values in OMC-1 suggest that most of these differences would reflect the stronger influence of gravity affecting the gas bulk motions within clustered regions (see Sect. 3.5).

The observed fiber lengths in massive filaments also appear to be smaller compared to those found at low-mass regimes. In Fig. 11 (right), we identify a systematic shortening of the fiber lengths as a function of the cloud mass. A two-sided
Kolmogorov-Smirnov test of the observed fiber lengths in Taurus and Orion indicates a negligible probability $\left(<10^{-4} \%\right)$ for these distributions to be drawn from the same sample. These differences are also apparent from the analysis of the fiber widths (see Sect. 3.4). Our ALMA data show a significant reduction of the observed fiber widths in dense environments like Orion $(F W H M \sim 0.035 \mathrm{pc})$ respect to those in low-mass clouds like Taurus or Musca ( $F W H M \sim 0.1 \mathrm{pc})$ (see Table 2). In combination with their transonic dynamical state, the proposed density dependency of these fiber properties (see Sect. 3.4) favors a scenario in which fibers likely emerge as part of a self-regulated process controlled by the thermal and density structures of their local environments.

The initial gas densities of the fibers may also set the conditions for local gravitational fragmentation. Along both OMC-1 (Teixeira et al. 2016) and OMC-2 (Kainulainen et al. 2017), interferometric studies have identified typical Jeans-like separations between embedded sources (cores \& protostars) of $\lambda_{\text {frag }}=0.06-0.08 \mathrm{pc}(12000-17000$ AU). These scales contrast with the typical inter-core distances in Taurus with $\lambda_{\text {frag }} \sim 0.3 \mathrm{pc}$ (60000 AU) (Tafalla \& Hacar 2015), that is, $\sim 3.5-5$ times larger than in Orion. Although apparently different, the above variations are in relatively good agreement with the expected density dependence of the (critical) fragmentation scales for hydrostatic filaments, in which $\lambda_{\text {crit }}=3.94 H$ or $\sim 2 \times F W H M$ (Stodólkiewicz 1963). In both Taurus and Orion, the observed fibers closely follow these predictions showing roughly similar $\frac{\lambda_{\text {frag }}}{F W H M} \sim 3$ (see similar results in Schneider \& Elmegreen 1979).

\subsection{Towards a unified model of star formation}

The detection of fibers in Orion opens a new window on the description of the internal gas structure in massive clouds. Since their discovery (Hacar et al. 2013), different studies have reported the detection of fibers in isolated and clustered starforming environments in clouds like Perseus (Hacar et al. 2017b) and Serpens (Lee et al. 2014; Fernández-López et al. 2014). Similar networks of small-scale filaments are apparent in other nearby regions like rho-Oph (André et al. 2007), B59 (Román-Zúñiga et al. 2009), and L1517 (Hacar \& Tafalla 2011). Our new ALMA 


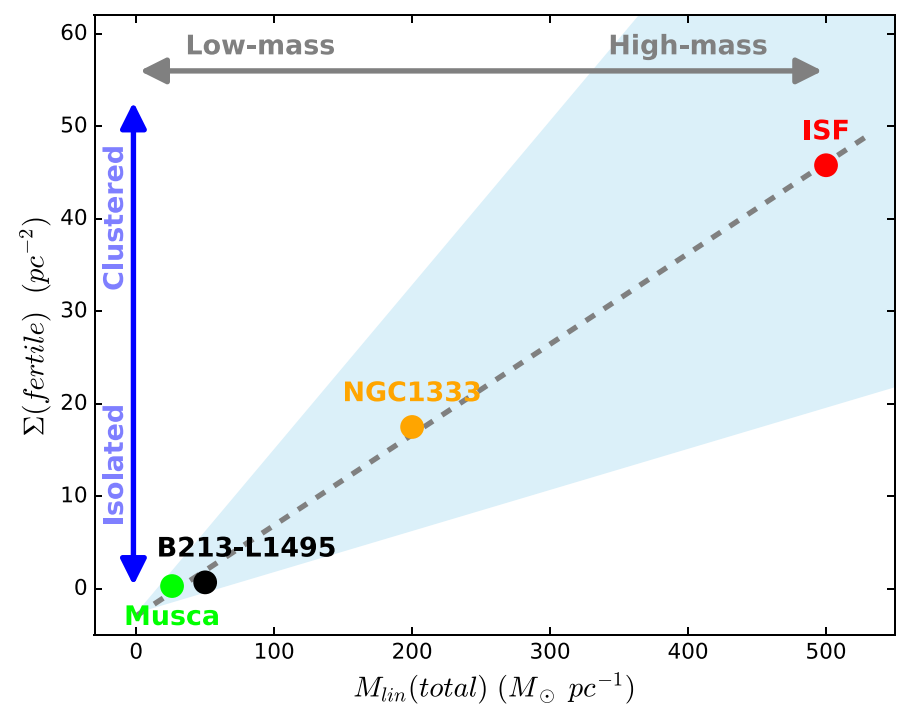

Fig. 12. Empirical correlation between the total filament mass per unit length $\left(\mathbf{M}_{\text {lin }}\right.$ (total)) and the obtained surface density of fertile fibers ( $\Sigma$ (fertile)) in Musca (green), B213-L1495 (black), NGC1333 (orange), and ISF (red). The dashed line indicates the least-square fit of the above four clouds for which $\Sigma$ (fertile) $\sim 0.1 \times \mathrm{M}_{\text {lin }}$ (total). The shaded blue area shows the expected variations of a factor of 2 respect to this linear correlation. Blue and gray arrows indicate the proposed tendency of these fiber networks to form either isolated (bottom) or clustered (top) stellar populations and both low- (left) and high-mass (right) stars.

observations demonstrate that this underlying organization is not restricted to low-mass clouds but is also intrinsic of regions at higher mass regimes. Forming networks of different complexity, these fibers dominate the internal gas substructure independently of the environment, stellar content, or initial cloud conditions. In all cases, this pre-existing fiber substructure is directly linked to the position of stars and cores, likely controlling their formation. The widespread detection of fibers seems to reflect the preferred organization mechanism for the dense molecular gas within clouds. While their origin remains a matter of debate (Smith et al. 2014; Moeckel \& Burkert 2015; Clarke et al. 2017; Smith et al. 2016), the formation of these transonic fibers appears to be an inherent property of the ISM turbulence and an essential ingredient of the star formation process in both low- and high-mass regions.

Hacar et al. (2017b) have proposed a correlation between the surface density of cores and protostars with the surface density of fertile fibers $(\Sigma$ (fertile) $)$. Under this hypothesis, a distributed population of stars would originate in clouds with an intrinsic low density of fertile fibers. On the other hand, more compact stellar systems would arise in densely populated fiber environments. Our observations extend these results towards massive clusters like the ONC. Originally postulated connecting lowmass regions like Taurus and Perseus, where $\frac{\Sigma(\text { fertile,Per) }}{\Sigma(\text { fertile,Tau) }} \sim 30$ (see Hacar et al. 2017b), we find increasing values of $\frac{\Sigma(\text { fertile,Ori) }}{\Sigma \text { (fertile,Tau) }} \sim$ 50-80 in comparison with Orion (see in Table 2). As shown in Fig. 12, these reported ratios consistently increase with the typical mass per unit length of these filamentary clouds $\left(\mathrm{M}_{\text {lin }}\right.$ (total)) showing a surprising linear trend approximately described by a least-square fit $\Sigma($ fertile $) \sim 0.1 \times \mathrm{M}_{\text {lin }}$ (total) $(\text { gray dashed line })^{5}$.

\footnotetext{
5 Local variations of a factor of $\sim 2$ in both $\mathrm{M}_{\text {lin }}$ (total) and $\Sigma$ (fertile) values are apparent in all Taurus, Perseus, and Orion regions (e.g., see values for OMC-1 and OMC-2 in Table 2) introducing similar uncertainties in this linear fit (see blue shaded area in Fig. 12).
}

The lack of a complete census of protostars and cores in OMC-1 hampers a direct comparison between these fiber densities and the current young stellar population of this cloud (see Hacar et al. 2017b, for a discussion). Nevertheless, the systematic increase of the fiber densities across two orders of magnitude suggests that the different star formation activity in these regions may be directly related to the intrinsic complexity of their fiber substructure.

The above variations of the fiber surface density could potentially unify our description of the star-formation process in isolated and clustered regions. In particular, Fig. 12 indicates a continuity between the different star-formation scenarios classically distinguished in the literature (see arrows in this figure). Rather than a distinct physical process (i.e., qualitative change), the origin of clusters in clouds like Orion would be then explained as a scaled-up version (i.e., quantitative change) of those low-mass, Taurus-like regions forming stars in isolation. Moreover, the detection of the above fiber networks introduces an observational link between low- and high-mass star-formation mechanisms. Individually fragmenting and forming low-mass cores, these fibers would likely collide and merge in compact networks promoting the formation of localized high-mass, superJeans overdensities (see also Hacar et al. 2017b). Interestingly, this emergent behavior would be naturally favored by the mutual gravitational attraction between fibers in densely populated environments explaining the formation of massive objects at the center of hub-like associations (Sect. 3.5).

The above unified fiber scenario provides a direct prediction for future observations. According to Fig. 12, highmass star-forming filaments with $\mathrm{M}_{\text {lin }}$ (total) $\gg 500 M_{\odot} \mathrm{pc}^{-1}$ are expected to be resolved into dense bundles of fibers in which $\Sigma$ (fertile) $\gtrsim 50 \mathrm{pc}^{-2}$. Following our results in Orion (see Sect. 3.4), these compact configurations would be possible if fibers continue reducing their physical dimensions in higher density regimes. Current observational evidence indicates increasing levels of substructure at sub-parsec scales in massive IRDCs (e.g., Peretto et al. 2014; Beuther et al. 2015; Henshaw et al. 2016). Additional high-resolution studies are needed to confirm these results in different galactic environments in young proto-clusters of increasing mass and complexity.

\section{Conclusions}

In this Paper I, we have investigated the dense gas substructure of the massive Integral Shape Filament in Orion using a new set of ALMA Cycle-3 observations. We have created a high-dynamic-range map of the $\mathrm{N}_{2} \mathrm{H}^{+}$(1-0) emission in the ISF combining two 148-pointing mosaics with previous large-scale IRAM 30m single-dish observations. From the detailed analysis of the gas kinematics, we have systematically characterized the internal organization and physical properties of the dense gas $\left(n\left(\mathrm{H}_{2}\right)>10^{5} \mathrm{~cm}^{-3}\right)$ within both OMC-1 and OMC-2 regions down to resolutions of $0.009 \mathrm{pc}$ (or $\sim 2000 \mathrm{AU}$ ). The results of this work are summarized as follows:

1. We conservatively identify a total of 55 velocity-coherent fibers along both OMC-1 and OMC-2 clouds. Forming a braided network, these fibers explain the complex substructure of the massive ISF as well as its current distribution of cores and protostars (Sect. 3). Systematically observed in regions like Musca, Taurus, Perseus, and Orion, our results highlight the importance of fibers as the fundamental dense components of both low- and high-mass filaments. 
2. Independently of the environment, the ISF fibers present transonic non-thermal velocity dispersions with respect to their local temperature (Sect. 3.2). Most of these fibers are largely supported by a combination of thermal and sonic-like turbulent motions showing a (sub-)critical mass distribution (Sect. 3.3). This quiescent dynamical state promotes the initial conditions for subsequent gravitational fragmentation. With analogous properties to those of low-mass regions, the detection of transonic fibers seems to reflect the preferred gas organization prior to the formation of stars.

3. The ISF fibers present a narrow radial distribution showing typical FWHM widths with a median value of $0.035 \mathrm{pc}$ (Sect. 3.4). This value is between approximately two and five times smaller than that found in regions like Perseus or Taurus. In addition to previous works, the statistical study of the ISF fibers demonstrates the existence of filamentary structures with compact radial distributions narrower than the so-called universal $0.1 \mathrm{pc}$ width.

4. Additionally, we observe a systematic shortening of the fibers between regions like Taurus and Orion (Sect. 3.4). Both width and length variations seem to have their origin in the significantly higher gas densities found in massive filaments $\left(n\left(\mathrm{H}_{2}\right)>10^{7} \mathrm{~cm}^{-3}\right)$ when compared to low-mass regions (i.e., $\left.n\left(\mathrm{H}_{2}\right) \sim 10^{5} \mathrm{~cm}^{-3}\right)$. This density dependency indicates that the physical dimensions of the fibers may be self-regulated by the initial gas conditions.

5. The ISF fibers are spatially organized in distinct hub-like associations. We find a tentative correlation between the complexity and global kinematics of these fiber networks with the total mass distribution within both OMC-1 and OMC-2. These results suggest that fibers are internally oriented following the local gravitational potential within the ISF (Sect. 3.5; see also Appendix B.1).

6. Finally, we find a systematic increase of the surface density of star-forming (fertile) fibers as a function of the total mass per unit length in filamentary regions like Musca, B213-L1495, NGC1333, and the ISF (Sect. 4.2). Based on this empirical correlation, we propose a unified star-formation scenario where the observational differences between both low- and high-mass clouds, as well as both isolated and clustered regimes, naturally emerge from their initial concentration of fibers.

Acknowledgements. The authors thank the anonymous referee for helping to improve the quality of the paper. AH thanks Alvaro Sanchez-Monge, Yanett Contreras, Daniel Harsono, and the ALLEGRO team for their support during the reduction of the ALMA data. AH also thanks Andreas Burkert and Ewine van Dishoeck for their insightful discussions and comments. This work is part of the research programme VENI with project number 639.041.644, which is (partly) financed by the Netherlands Organisation for Scientific Research (NWO). MT and AH thank the Spanish MINECO for support under grant AYA201679006-P. JG acknowledges funding by the Austrian Science Fund (FWF) under project number P 26718-N27. This paper makes use of the following ALMA data: ADS/JAO.ALMA\#2015.1.00669.S. PST acknowledges support from the STFC (grant number ST/M001296/1). ALMA is a partnership of ESO (representing its member states), NSF (USA) and NINS (Japan), together with NRC (Canada) and NSC and ASIAA (Taiwan) and KASI (Republic of Korea), in cooperation with the Republic of Chile. The Joint ALMA Observatory is operated by ESO, AUI/NRAO and NAOJ. Based on observations carried out with the IRAM $30 \mathrm{~m}$ Telescope. IRAM is supported by INSU/CNRS (France), MPG (Germany) and IGN (Spain). This research made use of APLpy, an open-source plotting package for Python (Robitaille \& Bressert 2012). This research made use of Astropy, a community-developed core Python package for Astronomy
(Astropy Collaboration 2013). This paper made use of the TOPCAT software (Taylor 2005).

\section{References}

André, P., Belloche, A., Motte, F., \& Peretto, N. 2007, A\&A, 472, 519 André, P., Men'shchikov, A., Bontemps, S., et al. 2010, A\&A, 518, L102

André, P., Di Francesco, J., Ward-Thompson, D., et al. 2014, Protostars and Planets VI (Tucson: University of Arizona Press), 27

André, P., Revéret, V., Könyves, V., et al. 2016, A\&A, 592, A54 Arzoumanian, D., André, P., Didelon, P., et al. 2011, A\&A, 529, L6

Arzoumanian, D., André, P., Peretto, N., \& Könyves, V. 2013, A\&A, 553, A119

Astropy Collaboration, Robitaille, T. P., Tollerud, E. J., et al. 2013, A\&A, 558, A33

Bally, J. 2008, in Handbook of Star Forming Regions, volume I: The Northern Sky, ed. B. Reipurth (ASP Monograph Publications), 4, 459

Bally, J., Langer, W. D., Stark, A. A., \& Wilson, R. W. 1987, ApJ, 312, L45

Bally, J., Cunningham, N. J., Moeckel, N., et al. 2011, ApJ, 727, 113

Barnard, E. E., Frost, E. B., \& Calvert, M. R. 1927, in A Photographic Atlas of Selected Regions of the Milky Way (Washington: Carnegie institution of Washington), 1927

Beaumont, C. N., Offner, S. S. R., Shetty, R., Glover, S. C. O., \& Goodman, A. A. 2013, ApJ, 777,173

Bergin, E. A., \& Tafalla, M. 2007, ARA\&A, 45, 339

Berné, O., Marcelino, N., \& Cernicharo, J. 2014, ApJ, 795, 13

Beuther, H., Ragan, S. E., Johnston, K., et al. 2015, A\&A, 584, A67

Bonnell, I. A., Larson, R. B., \& Zinnecker, H. 2007, Protostars and Planets V, 149

Buckle, J. V., Davis, C. J., Francesco, J. D., et al. 2012, MNRAS, 422, 521

Busquet, G., Zhang, Q., Palau, A., et al. 2013, ApJ, 764, L26

Caselli, P., Benson, P. J., Myers, P. C., \& Tafalla, M. 2002, ApJ, 572, 238

Chini, R., Reipurth, B., Ward-Thompson, D., et al. 1997, ApJ, 474, L135

Clarke, S. D., Whitworth, A. P., Duarte-Cabral, A., \& Hubber, D. A. 2017, MNRAS, 468, 2489

Dutrey, A., Duvert, G., Castets, A., et al. 1991, A\&A, 247, L9

Fehér, O., Tóth, L. V., Ward-Thompson, D., et al. 2016, A\&A, 590, A75

Fernández-López, M., Arce, H. G., Looney, L., et al. 2014, ApJ, 790, L19

Forbrich, J., Öberg, K., Lada, C. J., et al. 2014, A\&A, 568, A27

Forbrich, J., Rivilla, V. M., Menten, K. M., et al. 2016, ApJ, 822, 93

Friesen, R. K., Pineda, J. E., Rosolowsky, E., et al. 2017, ApJ, 843, 63

Furlan, E., Fischer, W. J., Ali, B., et al. 2016, ApJS, 224, 5

Galván-Madrid, R., Zhang, Q., Keto, E., et al. 2010, ApJ, 725, 17

Genzel, R., Ho, P. T. P., Bieging, J., \& Downes, D. 1982, ApJ, 259, L103

Getman, K. V., Flaccomio, E., Broos, P. S., et al. 2005, ApJS, 160, 319

Goddi, C., Greenhill, L. J., Humphreys, E. M. L., Chandler, C. J., \& Matthews, L. D. 2011, ApJ, 739, L13

Goicoechea, J. R., Teyssier, D., Etxaluze, M., et al. 2015, ApJ, 812, 75

Grosso, N., Feigelson, E. D., Getman, K. V., et al. 2005, ApJS, 160, 530

Hacar, A., \& Tafalla, M. 2011, A\&A, 533, A34

Hacar, A., Tafalla, M., Kauffmann, J., \& Kovács, A. 2013, A\&A, 554, A55

Hacar, A., Kainulainen, J., Tafalla, M., Beuther, H., \& Alves, J. 2016, A\&A, 587, A97

Hacar, A., Alves, J., Tafalla, M., \& Goicoechea, J. R. 2017a, A\&A, 602, L2

Hacar, A., Tafalla, M., \& Alves, J. 2017b, A\&A, 606, A123

Hartmann, L. 2002, ApJ, 578, 914

Hartmann, L., \& Burkert, A. 2007, ApJ, 654, 988

Heigl, S., Burkert, A., \& Hacar, A. 2016, MNRAS, 463, 4301

Hennemann, M., Motte, F., Schneider, N., et al. 2012, A\&A, 543, L3

Henning, T., Linz, H., Krause, O., et al. 2010, A\&A, 518, L95

Henshaw, J. D., Caselli, P., Fontani, F., Jiménez-Serra, I., \& Tan, J. C. 2014 MNRAS, 440, 2860

Henshaw, J. D., Caselli, P., Fontani, F., et al. 2016, MNRAS, 463, 146

Henshaw, J. D., Jiménez-Serra, I., Longmore, S. N., et al. 2017, MNRAS, 464, L31

Hill, T., Motte, F., Didelon, P., et al. 2011, A\&A, 533, A94

Ikeda, N., Sunada, K., \& Kitamura, Y. 2007, ApJ, 665, 1194

Inutsuka, S.-i., \& Miyama, S. M. 1997, ApJ, 480, 681

Jackson, J. M., Finn, S. C., Chambers, E. T., Rathborne, J. M., \& Simon, R. 2010, ApJ, 719, L185

Johnstone, D., \& Bally, J. 1999, ApJ, 510, L49

Kainulainen, J., Ragan, S. E., Henning, T.,\& Stutz, A. 2013, A\&A, 557, A120

Kainulainen, J., Hacar, A., Alves, J., et al. 2016, A\&A, 586, A27

Kainulainen, J., Stutz, A. M., Stanke, T., et al. 2017, A\&A, 600, A141

Kauffmann, J., Goldsmith, P. F., Melnick, G., et al. 2017, A\&A, 605, L5

Kounkel, M., Hartmann, L., Loinard, L., et al. 2014, ApJ, 790, 49

Kuznetsova, A., Hartmann, L., \& Burkert, A. 2017, ApJ, 836, 190 
A. Hacar et al. : An ALMA study of the Orion Integral Filament. I.

Lee, K. I., Fernández-López, M., Storm, S., et al. 2014, ApJ, 797, 76

Li, D., Kauffmann, J., Zhang, Q., \& Chen, W. 2013, ApJ, 768, L5

Li, G.-X., Urquhart, J. S., Leurini, S., et al. 2016, A\&A, 591, A5

Lombardi, M., Bouy, H., Alves, J., \& Lada, C. J. 2014, A\&A, 566, A45

Martin-Pintado, J., Rodriguez-Franco, A., \& Bachiller, R. 1990, ApJ, 357, L49

McMullin, J. P., Waters, B., Schiebel, D., Young, W., \& Golap, K. 2007, Astronomical Data Analysis Software and Systems XVI, 376, 127

Meingast, S., Alves, J., Mardones, D., et al. 2016, A\&A, 587, A153

Megeath, S. T., Gutermuth, R., Muzerolle, J., et al. 2012, AJ, 144, 192

Menten, K. M., Reid, M. J., Forbrich, J., \& Brunthaler, A. 2007, A\&A, 474, 515

Mezger, P. G., Zylka, R., \& Wink, J. E. 1990, A\&A, 228, 95

Moeckel, N., \& Burkert, A. 2015, ApJ, 807, 67

Molinari, S., Swinyard, B., Bally, J., et al. 2010, A\&A, 518, L100

Motte, F., Bontemps, S., \& Louvet, F. 2017, ArXiv e-prints [arXiv: 1706.00118]

Muench, A., Getman, K., Hillenbrand, L., \& Preibisch, T. 2008, in Handbook of Star Forming Regions, volume I: The Northern Sky, ed. B. Reipurth (ASP Monograph Publications), 4, 483

Myers, P. C. 2009, ApJ, 700, 1609

Myers, P. C., \& Benson, P. J. 1983, ApJ, 266, 309

O'dell, C. R. 2001, ARA\&A, 39, 99

O'Dell, C. R., Muench, A., Smith, N., \& Zapata, L. 2008, in Handbook of Star Forming Regions, volume I: The Northern Sky, ed. B. Reipurth (ASP Monograph Publications), 4, 544

Ostriker, J. 1964, ApJ, 140, 1056

Pagani, L., Daniel, F., \& Dubernet, M.-L. 2009, A\&A, 494, 719

Palau, A., Zapata, L. A., Roman-Zuniga, C. G., et al. 2017, ApJ, submitted [arXiv: 1706.04623]

Palmeirim, P., André, P., Kirk, J., et al. 2013, A\&A, 550, A38

Panopoulou, G. V., Psaradaki, I., Skalidis, R., Tassis, K., \& Andrews, J. J. 2017, MNRAS, 466, 2529

Peretto, N., \& Fuller, G. A. 2009, A\&A, 505, 405

Peretto, N., Fuller, G. A., Duarte-Cabral, A., et al. 2013, A\&A, 555, A112

Peretto, N., Fuller, G. A., André, P., et al. 2014, A\&A, 561, A83

Peterson, D. E., \& Megeath, S. T. 2008, in Handbook of Star Forming Regions, volume I: The Northern Sky, ed. B. Reipurth (ASP Monograph Publications), 4,590
Pety, J., Guzmán, V. V., Orkisz, J. H., et al. 2017, A\&A 599, A98 Pineda, J. E., Goodman, A. A., Arce, H. G., et al. 2011, ApJ, 739, L2

Rivilla, V. M., Martín-Pintado, J., Sanz-Forcada, J., Jiménez-Serra, I., \& Rodríguez-Franco, A. 2013, MNRAS, 434, 2313

Robitaille, T., \& Bressert, E. 2012, Astrophysics Source Code Library [record ascl: 1208.017]

Rodriguez-Franco, A., Martin-Pintado, J., Gomez-Gonzalez, J., \& Planesas, P. 1992, A\&A, 264, 592

Román-Zúñiga, C. G., Lada, C. J., \& Alves, J. F. 2009, ApJ, 704, 183

Salji, C. J., Richer, J. S., Buckle, J. V., et al. 2015, MNRAS, 449, 1782

Schisano, E., Rygl, K. L. J., Molinari, S., et al. 2014, ApJ, 791, 27

Schneider, S., \& Elmegreen, B. G. 1979, ApJS, 41, 87

Schneider, N., Csengeri, T., Bontemps, S., et al. 2010, A\&A, 520, A49

Shimajiri, Y., Kawabe, R., Takakuwa, S., et al. 2011, PASJ, 63, 105

Shimajiri, Y., Kitamura, Y., Saito, M., et al. 2014, A\&A, 564, A68

Smith, R. J., Glover, S. C. O., \& Klessen, R. S. 2014, MNRAS, 445, 2900

Smith, R. J., Glover, S. C. O., Klessen, R. S., \& Fuller, G. A. 2016, MNRAS, 455, 3640

Stodólkiewicz, J. S. 1963, Acta Astron., 13, 30

Stutz, A. M., \& Kainulainen, J. 2015, A\&A, 577, L6

Stutz, A. M., Tobin, J. J., Stanke, T., et al. 2013, ApJ, 767, 36

Subrahmanyan, R., Goss, W. M., \& Malin, D. F. 2001, AJ, 121, 399

Tafalla, M., \& Hacar, A. 2015, A\&A, 574, A104

Takahashi, S., Ho, P. T. P., Teixeira, P. S., Zapata, L. A., \& Su, Y.-N. 2013, ApJ, 763,57

Tatematsu, K., Kandori, R., Umemoto, T., \& Sekimoto, Y. 2008, PASJ, 60, 407

Taylor, M. B. 2005, Astronomical Data Analysis Software and Systems XIV, 347,29

Teixeira, P. S., Takahashi, S., Zapata, L. A., \& Ho, P. T. P. 2016, A\&A, 587, A47

van der Tak, F. F. S., Black, J. H., Schöier, F. L., Jansen, D. J., \& van Dishoeck, E. F. 2007, A\&A, 468, 627

Vázquez-Semadeni, E., González-Samaniego, A., \& Colín, P. 2017, MNRAS, 467, 1313

Wiseman, J. J., \& Ho, P. T. P. 1998, ApJ, 502, 676

Zamora-Avilés, M., Ballesteros-Paredes, J., \& Hartmann, L. W. 2017, MNRAS, 472,647 


\section{Appendix A: Fiber identification in high-dynamic-range emission maps}

As a differentiating characteristic, fibers have been observationally defined as velocity-coherent structures identified by their spatial and velocity continuity. Exploiting this property in large molecular line datasets, Hacar et al. (2013) created the FriendIn-Velocity (FIVE) analysis technique. Compared to the direct analysis of the full 3D Position-Position-Velocity (PPV) analysis carried out by previous algorithms, FIVE combines a distinct $(1+3 \mathrm{D})$ treatment of the spectral data cubes. In a first stage, FIVE characterizes the individual kinematic properties of each gas component (e.g., centroid velocity $V_{l s r}$, line full-width-athalf-maximum $\Delta V$, peak intensity, and integrated area) extracted from the spectra (1D) using a supervised fitting technique. After that, FIVE investigates the (3D) continuity of the previously extracted components from the analysis of their line centroids in the PPV space using a unique velocity gradient $\nabla V_{l s r, 0}$ as linking parameter between nearby points. Connecting both velocity and spatial dimensions, this secondary process is carried out in two steps. FIVE firstly attempts to reconstruct the main skeletons of each individual structure from a selected subset of points with line intensities above a certain threshold $\mathrm{I}_{0}$ surrounded by a given number $\mathrm{N}_{\text {friends }}$ of PPV companions of similar properties. Once identified, FIVE uses these initial seeds to associate the rest of components following the same linking criteria (see Hacar et al. 2013, for additional details).

FIVE has been successfully employed on the characterization of fibers in the B213-L1495 (FIVE v1.0; Hacar et al. 2013, $\theta_{F W H M}=0.040 \mathrm{pc}$ ) and NGC 1333 (FIVE v1.1; Hacar et al. $2017 \mathrm{~b}, \theta_{F W H M}=0.035 \mathrm{pc}$ ) star-forming regions. In both cases, this FIVE analysis has benefited from the smooth velocity structure and the relatively small intensity variations observed within these clouds, allowing a direct identification of their fiber population using a single combination of $\left[\nabla V_{l s r, 0}, \mathrm{I}_{0}, \mathrm{~N}_{\text {friends }}\right]$ values. Although multiple velocity coherent structures are clearly identified in our spectra, this simplified criterion is, however, challenged by the large intensity variations and the complex velocity structure found in Orion (see also Appendix B). A series of individual tests indicate that no single combination of $\left[\nabla V_{l s r, 0}, \mathrm{I}_{0}, \mathrm{~N}_{\text {friends }}\right]$ values is able to satisfactorily recover all the structures identified in our $\mathrm{N}_{2} \mathrm{H}^{+}$integrated intensity maps in both OMC-1 and OMC-2 regions simultaneously (see Fig. 2). The association in velocity carried out by FIVE is also hindered by the presence of highly variable velocity gradients at large-scales, dominating the kinematic structure of the OMC-1 region (see Hacar et al. 2017a). Moreover, both intensity and velocity changes present a strong variability showing rapid changes at scales comparable to our ALMA beam size $\left(\theta_{F W H M} \sim 0.009 \mathrm{pc}\right)$, that is, more than four times smaller than in any previous work. In the light of the above, a new approach is needed to investigate our high-dynamic range emission maps in Orion.

Motivated by the observed emission properties of the ISF (Sect. 3), we have created a new hierarchical version of FIVE, hereafter referred to as HiFIVE. This new HiFIVE analysis introduces three main variants to the original FIVE identification scheme optimizing the segmentation of hierarchically related substructures in clouds with large internal velocity variations. First, the initial line intensity $I_{0}$ threshold is replaced by a selection criterion based on the total gas column density $W_{i}$ (see the conversion between $\mathrm{N}_{2} \mathrm{H}^{+}$intensities and total column densities in Sect. 3). Second, the former linking parameter $\nabla V_{l s r, 0}$ is substituted by an adaptive velocity gradient $\nabla V_{l s r, i}=\frac{1}{2} \frac{\Delta V_{i}}{\theta_{F W H M}}$, self-defined from the local line full-width-at-half-maximum $\Delta V_{i}$ and the beam size $\theta_{F W H M}$. Otherwise arbitrarily determined, this latter choice introduces a physically motivated definition of the linking parameter based on the resolution criterion by Nyquist in which two Gaussian lines can only be distinguished if their centroids are separated by $\delta V_{i} \geq \Delta V_{i} / 2$. Third, and as new feature, HiFIVE carries out a hierarchical segmentation in three recursive levels $\left(W_{i}, N_{i}\right)(\mathrm{i}=1,2,3)$ following a bottom-up approach. For that, the identification algorithm is first applied to the full dataset identifying the main kinematic structures (trees) recovered using a set of predefined $W_{1}$ and $N_{1}$ threshold. After that, a new algorithm execution is then independently carried out in each of these kinematic substructures identifying those separated fragments (branches) defined by a new selection criterion $W_{2}>$ $W_{1}$ and $N_{2} \leq N_{1}$. Finally, a third level of refinement (leaves) is then run for each of the previous new fragments with more than one independent substructure satisfying an intensity cut such that $W_{3}>W_{2}>W_{1}$ and $N_{3} \leq N_{2} \leq N_{1}$. The updates introduced in this new HiFIVE version reduce the selection parameters to the definition of the $\left(W_{i}, N_{i}\right)$ threshold pairs while the kinematic link between points is self-defined and locally adapted by the algorithm.

We have investigated the performance of HiFIVE with a reanalysis of the $\mathrm{N}_{2} \mathrm{H}^{+}$emission in NGC1333 (see also Hacar et al. 2017b). Different tests indicate that the majority of the velocity-coherent fibers recovered by HIFIVE $(\sim 70 \%)$ are identified in the first stage of the algorithm (aka trees), while subsequent refinement levels (branches and leaves) help to disentangle some of the internal substructure in the most complex objects. As a rule of thumb, lower $\left(W_{i}, N_{i}\right)$ pairs lead into higher levels of fragmentation. The a priori arbitrary (and methoddependent) HiFIVE parameters suggests an statistical approach for the selection of the absolute $\left(W_{i}, N_{i}\right)$ thresholds. We thus adopted $W_{1}, W_{2}$, and $W_{3}$ values coincident with the $\sim 50 \%$ (median), 75\% (third quartile), and 90\% column densities values measured in $\mathrm{N}_{2} \mathrm{H}^{+}$within this region and a similar number of friends $N_{1}=N_{2}=N_{3}=10$. With this parameter choice, HiFIVE automatically recovers a total of 10 velocity-coherent structures within NGC1333, that is, 4 less than the 14 fibers identified by Hacar et al. (2017b) using a dedicated FIVE (v1.1) version. Differences in the total number of structures are created by the ambiguous assignment of components into fibers in regions with strong line multiplicity and compact velocity fields. Distinct merging and/or fragmentation effects are also observed by the different identification schemes used by these two algorithm versions. Similar conclusions are drawn from the reanalysis of the B213-L1495 region although the lower sensitivity of the spectra used in that study together with the inclusion of an additional tracers like $\mathrm{C}^{18} \mathrm{O}$ hampers a more detailed comparison. Overall, HiFIVE identifies a lower number of structures due to the larger linking gradients defined in regions showing lines with greater $\Delta V_{i}$ (see the connection between $\Delta V_{i}$ and $\nabla V_{l s r, i}$ above). Quantitative variations of $\sim 40 \%$ are thus expected in the estimated surface density of fibers $\Sigma$ (fertile) compared to the original FIVE. These limitations of the new HiFIVE version in complex regions are compensated by the enhanced adaptability of the new algorithm to the strong velocity variations in massive clouds (see Appendix B.2). Despite their differences, the mean fiber properties recovered in both HiFIVe and FIVE analysis (i.e., $\langle L\rangle,\left\langle\sigma_{N T} / \mathrm{c}_{s}\right\rangle,\left\langle\delta V_{L S R}\right\rangle$, etc) appear to be consistent within $1-\sigma$ level. While the individual assignment of components into different structures may vary depending on the algorithm version, our tests validate the statistical comparison of the new HiFIVE results with the analysis presented in previous studies. 

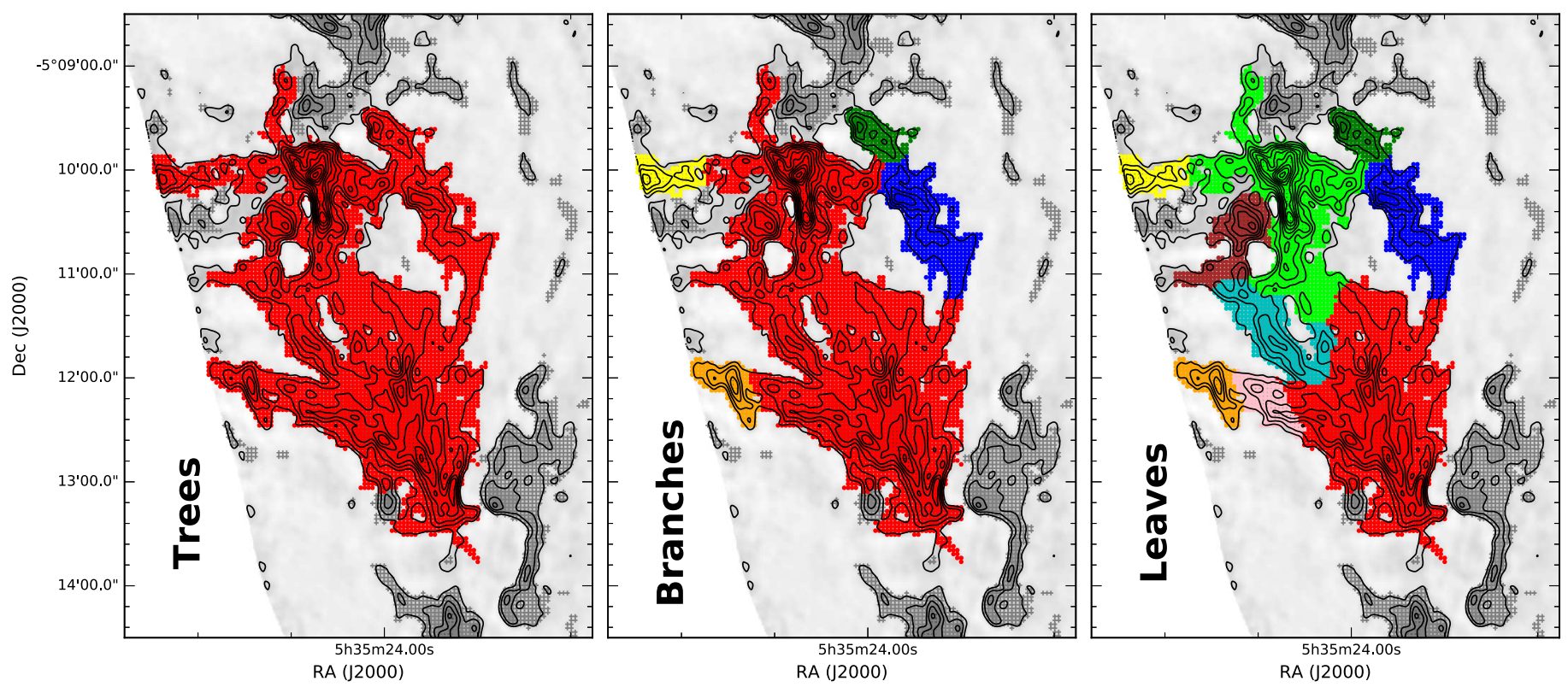

Fig. A.1. Structure identification in the OMC-2 region at different stages of the HiFIVE analysis. From left to right: (a) Trees, (b) Branches, and (c) Leaves (see text for a description). For simplicity, only those velocity structures extracted from the same initial tree are displayed in colors. The rest of the positions analyzed by HiFIVE are indicated by gray crosses. We notice that, although satisfactory in most cases, our HiFIVE analysis fails to describe some of the undoubtedly more complex gas substructure found in the ISF (e.g., fiber \#37, see central leave shown in red).

Figure 4 (right) shows the results of the HiFIVE analysis carried our in the ISF. Our analysis includes the (hyperfine) fit of $\sim 70000$ ALMA $\mathrm{N}_{2} \mathrm{H}^{+}$spectra from which we effectively extracted a total of $>25000$ individual components with $S / N \geq 3$ (Appendix B). Similar to NGC1333, we define the internal structure of the ISF using three hierarchical levels determined by equivalent column density thresholds of $W_{1}=2.3 \times$ $10^{22} \mathrm{~cm}^{-2}$ (or $\sim 26 \mathrm{~A}_{v}$; median), $W_{2}=4.0 \times 10^{22} \mathrm{~cm}^{-2}$ (or $\sim 44 \mathrm{~A}_{v}$; third quartile), and $W_{3}=6.0 \times 10^{22} \mathrm{~cm}^{-2}$ (or $\sim 66 \mathrm{~A}_{v}$; $90 \%$ quantile) detected in our $\mathrm{N}_{2} \mathrm{H}^{+}$spectra (see Sect.3), all with $\mathrm{N}_{i}=10$. As result of this analysis, HiFIVE identifies a total of 55 velocity-coherent substructures (fibers) along the ISF. The above parameter selection satisfactorily recovers most of the detected emission along this cloud assigning $95 \%$ of the fitted components into fibers.

For visualization purposes, in Fig. A.1 we illustrate the performance of the HiFIVE algorithm characterizing the gas substructure in the OMC-2 region. Overall, we find an excellent correspondence between our HiFIVE velocity analysis and the structures identified in both ALMA- $\mathrm{N}_{2} \mathrm{H}^{+}$(Fig. 4, right) and SCUBA maps (Fig. 4, left). Consistent with our previous tests, an inspection by eye of the above results indicate that HiFIVE fails to decompose several of the most complex structures within our maps. Examples of this behavior can be found in the OMC-1 Ridge (fiber \#21), the OMC-2 FIR 6 cloud (fiber \#36), and the OMC-2 FIR 4 region (fiber \#43), all showing an additional complexity with multiple sub-branches not captured in our HiFIVE analysis (see Figure A.1c). Additional filamentary features not recovered HiFIVE are apparent at low intensities (e.g., see several fiber-like structures at the west side of the OMC-2 region running in parallel to the main ISF axis). Given the level of complexity observed in our $\mathrm{N}_{2} \mathrm{H}^{+}$maps, the above 55 fibers should be interpreted as a simplified description of the real dense gas substructure within the ISF.

The reconstruction analysis carried out by our HiFIVE algorithm assumes the direct correspondence between the continuous structures identified in the observed Position-Position-Velocity
(PPV) space and the real density structure of the PositionPosition-Position (PPP) space. Using simulations of clouds in global gravitational collapse, Zamora-Avilés et al. (2017) have recently suggested that some of the reconstructed fibers in the PPV space do not necessarily translate into physically coherent structures in PPP (see also Moeckel \& Burkert 2015, for similar conclusions from a different suite of simulations). Widely discussed in the past (e.g., see Beaumont et al. 2013, and references therein), Zamora-Avilés et al. (2017) remark how 1.o.s. superpositions and projection effects can affect the observational identification of different gas structures in molecular clouds (see also Smith et al. 2016). While potentially problematic in molecular line observations, these contamination effects seem to have a limited impact in our ALMA data in Orion. Those problematic cases found by Zamora-Avilés et al. correspond to low-mass fibers identified in regions with complex superpositions. Already in their simulations, however, the connection between both PPV and PPP spaces greatly improves in more massive and denser fibers. About two orders of magnitude larger than those found by Zamora-Avilés et al. (2017), the detection of fibers in Orion at densities of $\gtrsim 10^{7}-10^{8} \mathrm{~cm}^{-3}$ (Sect. 3) suggests a good correspondence with the mass distribution in the ISF. Reassuringly, most of the ISF fibers are also clearly separated in our emission maps (see Fig. 4). With perhaps the exception of some dubious cases in our maps (see Appendix B), we therefore expect a close agreement between the fibers recovered by our HiFIVE analysis and the true gas substructure of the ISF.

\section{Appendix B: Line decomposition}

The complexity of the gas velocity field observed in our Orion ALMA data requires a careful description of the line decomposition technique. In this Appendix, we describe the detailed line fitting procedure (Appendix B.1) as well as the main line properties derived from this analysis (Appendices B.2 and B.3). 


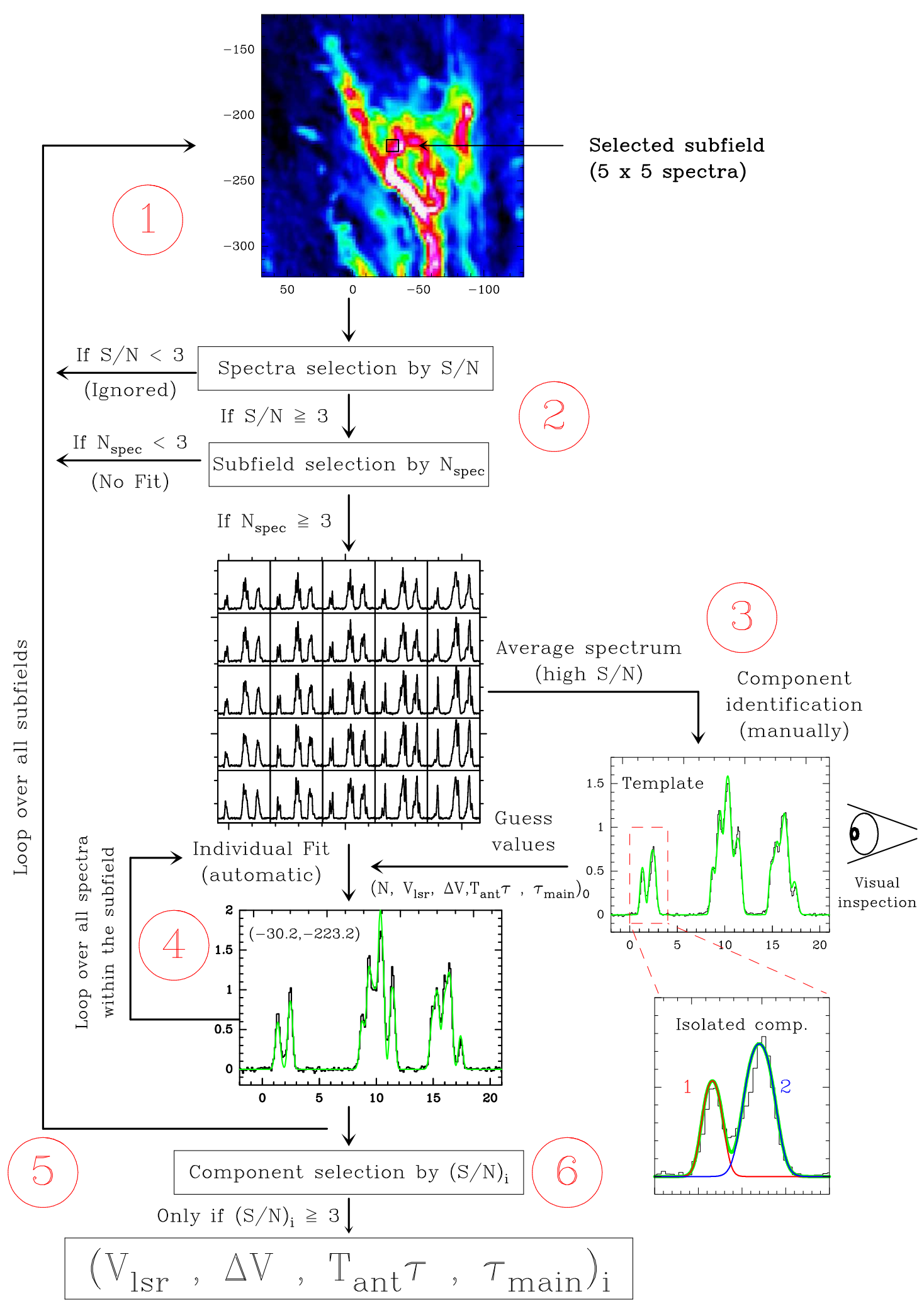

Fig. B.1. Workflow diagram describing the line fitting procedure of our $\mathrm{N}_{2} \mathrm{H}^{+}$(1-0) ALMA data in Orion. The different steps are numbered in red. See text for a description. 


\section{B.1. Fitting procedure}

Our spectral analysis adapts the fitting techniques introduced by Hacar et al. (2013) to the case of lines with hyperfine structure like $\mathrm{N}_{2} \mathrm{H}^{+}$. Our fitting strategy combines both semi-automatic and supervised approaches for the analysis of large molecular datasets. Figure B.1 illustrates the workflow describing the full fitting procedure summarized in the following six steps:

1. Subfield definition: The entire dataset is subdivided into subfields of $5 \times 5$ spectra. The size of this subfield is estimated from the region in which the main properties of the individual spectra (number of components, mean velocity, etc) do not change significantly across the field.

2. Selection: Within each subfield, a spectrum is only considered if it contains (at least) one emission channel above the mean rms in our dataset. A subfield is discarded if it does not contain at least three spectra fulfilling the above requirement.

3. Subfield template: All accepted spectra are averaged to obtain a high $\mathrm{S} / \mathrm{N}$ spectrum meant to describe the mean line properties within the subfield. This spectrum template is visually inspected and manually fitted. The number of components and the goodness of the fit are evaluated from the direct inspection of the isolated, optically thin $\left(J F_{1} F\right)=$ $(101-012)$ hyperfine component. If necessary, individual spectra are examined in order to refine this fit.

4. Automatic fit: The fitting results obtained in the previous step are used as guess values for the automatic fit of all accepted spectra within the subfield. Although informed in their initial values, all fitting parameters are left unconstrained.

5. Iteration: Steps 1-4 are repeated over all the subfields in our maps.

6. Component selection: A final $\mathrm{S} / \mathrm{N}$ selection is carried out for every individual component with respect to the rms level in each spectrum. Only velocity components with individual peak intensities with $S / N \geq 3$ in their central hyperfine $\left(J F_{1} F\right)=(123-012)$ line are accepted for their analysis.

The entire fitting procedure is carried out with the GILDAS/CLASS software ${ }^{6}$. All hyperfine lines are simultaneously fitted using the HFS method assuming similar excitation conditions and widths, as well as Gaussian opacity profiles for all components in the multiplet (i.e. steps $3 \& 4$ ). If resolved in the isolated $\left(J F_{1} F\right)=(101-012)$ hyperfine component, the presence of multiple peaks in our spectra, are assumed to correspond to independent gas structures and are, therefore, fitted independently (see an example in Fig. B.1). In case of doubt, the spectra are conservatively fitted with the lowest possible number of components. Non-Gaussian components producing self-absorbed profiles or line wings can potentially contaminate this analysis. Although these effects are identified in some localized spectra, the visual inspection of the data indicates a statistically small influence in our large dataset.

In Fig. B.2 we display a series of representative spectra fitted in our ALMA data. This figure includes both the total (Col. 1) plus individual line profiles (Cols. 2 and 3) as well as their residual emission after the subtraction of their best-fit solution (Col. 4). Panels a and b show the most common situations found in our spectra with either single or multiple well-resolved

6 http://www.iram.fr/IRAMFR/GILDAS components with velocity differences larger than the individual linewidth, that is, $\delta V_{l s r}>\Delta V$. In these simple cases, small residuals (few percent of the total integrated emission) are created by the slightly different excitation temperatures of each independent hyperfine component not considered in our fits. Panel c illustrates a close superposition of components denoting the limiting case where two superposed lines can be resolved if $\delta V_{l s r} \gtrsim \Delta V / 2$. For comparison, panel d illustrates an example of the problematic cases found in regions with extreme variability in velocity (i.e., not well described by their subfield template spectrum), unresolved multiplicity (e.g., too close to be separated in all spectra within the subfield), and/or with complex line profiles leading to poor or dubious fitting solutions (see individual components in this panel). Most of these last pathological effects are minimized by the conservative selection of components carried out in our analysis. Estimated to affect $<5 \%$ of our fits, their results are included in our kinematic analysis in order to preserve the total line intensity ${ }^{7}$.

Using the above technique, we analyzed more than 70000 $\mathrm{N}_{2} \mathrm{H}^{+}$(1-0) spectra in Orion. A total of $>25000$ individual components are detected with $S / N \geq 3$. For each of these components, the HFS method provides with the best-solution estimates for the line central velocity $V_{L S R}$, linewidth $\Delta V$, the intensity product $T_{\text {ant }} \tau$, and the total multiplet opacity $\tau_{\text {main }}$ from which all line properties can be derived ${ }^{8}$. Among these quantities, both $V_{L S R}$ and $\Delta V$ are estimated within typical errors of $0.01 \mathrm{~km} \mathrm{~s}^{-1}$. With significantly less accuracy, median relative uncertainties of $\sim 25 \%$ are measured in our $\tau_{\text {main }}$ estimates.

\section{B.2. Fitting results (I): kinematics}

In Fig. B.3 (first and second panels), we display the centroid velocities $V_{L S R}\left(\mathrm{~N}_{2} \mathrm{H}^{+}\right)$of all the components fitted in our $\mathrm{N}_{2} \mathrm{H}^{+}$ (1-0) spectra with $S / N \geq 3$ as function of declination along the ISF. Overall, the gas velocity structure reproduces the results obtained by previous single-dish observations. Along the $\sim 1.5 \mathrm{pc}$ of the OMC-2 region, the average gas velocity is dominated by a smooth north-south velocity gradient of $\sim 1 \mathrm{~km} \mathrm{~s}^{-1} \mathrm{pc}^{-1}$ (e.g., Bally et al. 1987). Closer to the ONC, the observed gas velocities define a (blue-shifted) $\mathrm{V}$-shape profile consistent the gravitational collapse of the OMC-1 region (Hacar et al. 2017b). These gravitationally induced motions generate the largest velocity differences along the ISF with maximum velocity shifts of $\sim 5 \mathrm{~km} \mathrm{~s}^{-1}$ in the surroundings of OMC-1 South. Although largely dominated by the stellar component, the bottom of the potential locates the current center of mass of the OMC-1 system (i.e. gas + stars) $\sim 60^{\prime \prime}$ away from the Trapezium. An additional kinematic complexity is revealed by ALMA at subparsec resolutions. Organized velocity fluctuations are found in the proximity of OMC-1 Ridge, OMC-2 FIR-4, and OMC-2 FIR-6. Superposed on them, a large variety of velocity gradients, with intensities between $1-20 \mathrm{~km} \mathrm{~s}^{-1} \mathrm{pc}^{-1}$, are seen at scales of $\sim 0.1-$ $0.3 \mathrm{pc}$. In more detail, several velocity-coherent structures can be directly identified as groups of continuous velocity in this plot.

Similar to the gas velocities, in Fig. B.3 (third panel) we illustrate the distribution of the $\mathrm{N}_{2} \mathrm{H}^{+}$linewidths $\left(\Delta V\left(\mathrm{~N}_{2} \mathrm{H}^{+}\right)\right)$ in both OMC-1 and OMC-2 clouds. Across the entire ISF, 75\%

\footnotetext{
7 We note that fits similar to the one shown in panel d (with $\delta V_{l s r} \lesssim$ $\Delta V / 2$ ) are likely recovered by HiFIVE into a single structure in velocity (see Appendix A). With little impact on the derived kinematic properties of fibers the addition of these components allows us to recover the complete line emission.

8 (see CLASS cookbook; https : //www . iram . fr/IRAMFR/GILDAS/ doc/pdf/class.pdf)
} 


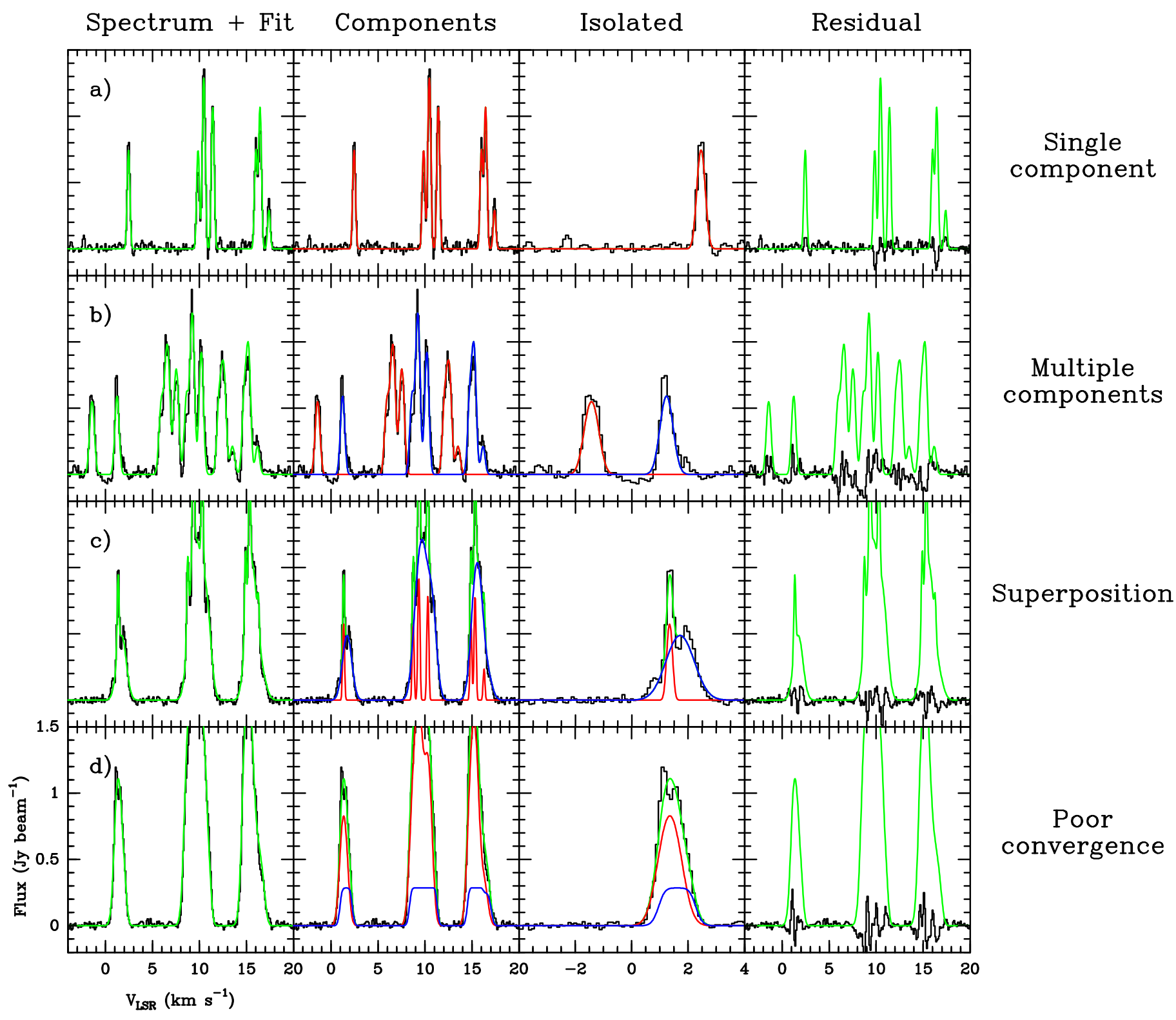

Fig. B.2. Examples of the $\mathrm{N}_{2} \mathrm{H}^{+}$(1-0) ALMA spectra found in Orion: from left to right: (1) observed spectrum and best-fit solution: (2) individual components identified by the fit; (3) zoom-in on the $\left(J F_{1} F\right)=(101-012)$ hyperfine component; (4) residual spectrum after the subtraction of the best-fit solution. For comparison, the best-fit solution (either single or multiple) is displayed in all subpanels in green. Each individual velocity component is denoted in red and blue in panels 2 and 3. From top to bottom: (a) single component; (b) well-resolved multiple components; (c) superposition of components; (d) problematic spectra.

of the observed linewidths show $\Delta V\left(\mathrm{~N}_{2} \mathrm{H}^{+}\right)<0.71 \mathrm{~km} \mathrm{~s}^{-1}$. We identify a systematic increase of the observed linewidths between OMC-2 $\left(\left\langle\Delta V\left(\mathrm{~N}_{2} \mathrm{H}^{+}\right)\right\rangle=0.48 \mathrm{~km} \mathrm{~s}^{-1}\right)$ and OMC-1 $\left(\left\langle\Delta V\left(\mathrm{~N}_{2} \mathrm{H}^{+}\right)\right\rangle=0.72 \mathrm{~km} \mathrm{~s}^{-1}\right)$ partially correlated with the increasing gas kinetic temperatures measured in the proximity of the ONC (see also forth panel). Localized line broadening effects are also found at the position of most of the embedded FIR sources (e.g., OMC-2 FIR-4) and several of the embedded protostars within both OMC-1 and OMC-2 regions (see spikes in the $\Delta V$ distributions).

The reported line emission properties within the ISF highlight some of the challenges investigating the internal velocity field of massive filaments. The characterization of these lines must consider the strong spatial variability observed in both velocity centroids and linewidths at the current ALMA resolution. Moreover, their analysis needs to evaluate the combined influence of all global and local motions in combination with feedback effects. These findings have motivated the development of our new algorithm HiFIVE (see Appendix A).

\section{B.3. Fitting results (II): line opacities}

In addition to the line kinematics, our fits provide detailed information of the total $\mathrm{N}_{2} \mathrm{H}^{+}(1-0)$ opacity $\left(\tau_{\text {main }}\right)$ as well as the corresponding opacities for each of its individual hyperfine components (with $\tau_{i}=r_{i} \cdot \tau_{\text {main }}$ for an hyperfine line of relative intensity $r_{i}$ ). We have evaluated the opacity of the extracted $\mathrm{N}_{2} \mathrm{H}^{+}$ $(1-0)$ fits from the analysis of the central $\left(J F_{1} F\right)=(123-012)$ transition $\tau(123-012)$, that is, the brightest and most optically thick hyperfine line in the multiplet $\left(r_{i}=7 / 27\right)$ and the one subject of largest saturation effects. Among all the extracted components, $\sim 75 \%$ show central line opacities with 

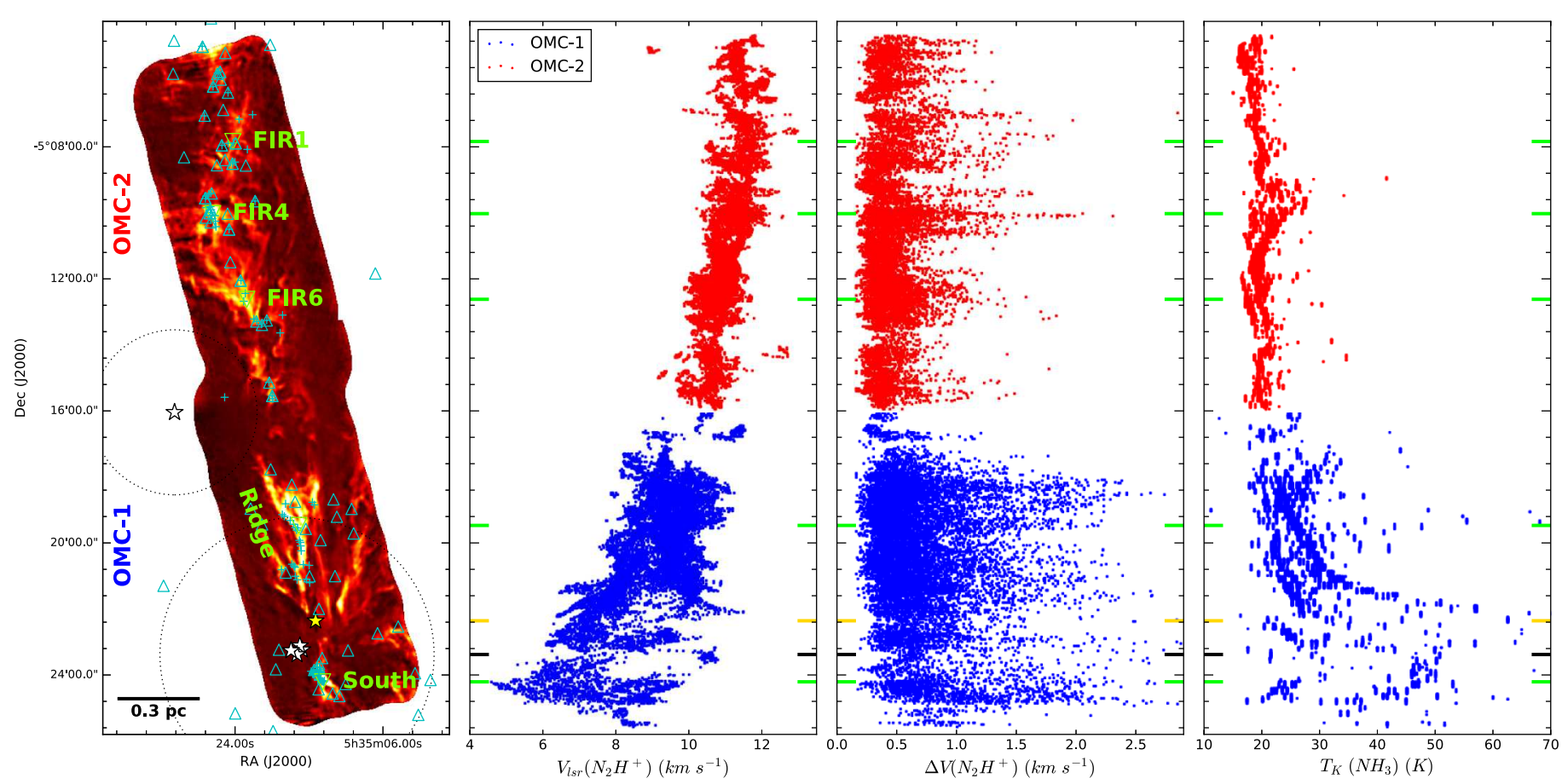

Fig. B.3. Gas kinematic properties as a function of declination along both OMC-1 (blue) and OMC-2 (red) regions. from left to right: (1) integrated $\mathrm{N}_{2} \mathrm{H}^{+}$(1-0) emission. Symbols are similar to those in Fig. 2; (2) centroid velocities and (3) linewidths for all the $\mathrm{N}_{2} \mathrm{H}^{+}(1-0)$ components detected in our ALMA observations with $S / N \geq 3$; (4) $\mathrm{NH}_{3}$-derived gas kinetic temperatures (Friesen et al. 2017) at the position of our $\mathrm{N}_{2} \mathrm{H}^{+}$measurements. For reference, the declination of the Trapezium stars (black), the Orion BN/KL region (yellow), and FIR sources (green) are indicated in all subplots.

$\tau(123-012)<1$ (optically thin) and $92 \% \tau(123-012)<2$ (moderate opacity). The other $8 \%$ include both high opacity spectra as well as problematic fits (see Appendix B.1). Most of these optically thick components are located within unresolved regions like OMC-1 Ridge, OMC-2 FIR 6 and OMC-2 FIR 4. In comparison, $99 \%$ of the isolated hyperfine $\left(J F_{1} F\right)=$ $(101-012)$ lines $\left(r_{i}=1 / 27\right)$ are estimated to be optically thin.

Figure B.4 shows our investigation of the correlation between the observed $\left(J F_{1} F\right)=(123-012)$ line opacities and the total $\mathrm{N}_{2} \mathrm{H}^{+}$column densities $\left(N\left(\mathrm{~N}_{2} \mathrm{H}^{+}\right)\right)$in the ISF. We have estimated these latter $N\left(\mathrm{~N}_{2} \mathrm{H}^{+}\right)$values from the individual $\tau_{\text {main }}$ and $T_{a n t} \tau$ line parameters following the standard approach introduced by Caselli et al. (2002). Using a kernel density estimate, this figure summarizes the results of more than 25000 components measured in our ALMA observations. We have compared our estimates with the radiative transfer predictions provided by RADEX (van der Tak et al. 2007). On average, the observed $\mathrm{N}_{2} \mathrm{H}^{+}$optically thin lines are consistent with the expected emission properties for the gas in the ISF (Sect. 3). In particular, the relatively low opacities detected in our $\mathrm{N}_{2} \mathrm{H}^{+}(1-0)$ spectra can be explained by the higher temperatures (20-30 K) and densities $\left(\sim 10^{7}-10^{8} \mathrm{~cm}^{-3}\right)$ found in Orion compared to the typical gas conditions in low-mass regions like Taurus (see models with $10 \mathrm{~K}$ and $10^{5} \mathrm{~cm}^{-3}$ ) leading to the depopulation of the $\mathrm{J}=1$ level in favour of those at higher energies $(\mathrm{J} \geq 3)$.

High line opacities can crucially affect the mass estimates derived from the integrated emission of tracers like $\mathrm{N}_{2} \mathrm{H}^{+}$. Opacity effects can hide large column densities in the case of heavily thick lines. Our statistical analysis demonstrates that these effects are minimized in the case of Orion. Based on these results, we adopted an optically thin approximation for all our mass estimates (Sect. 3). Strictly speaking, this assumption is valid in $75 \%$ of our measurements. Additional tests indicate a maximum (and

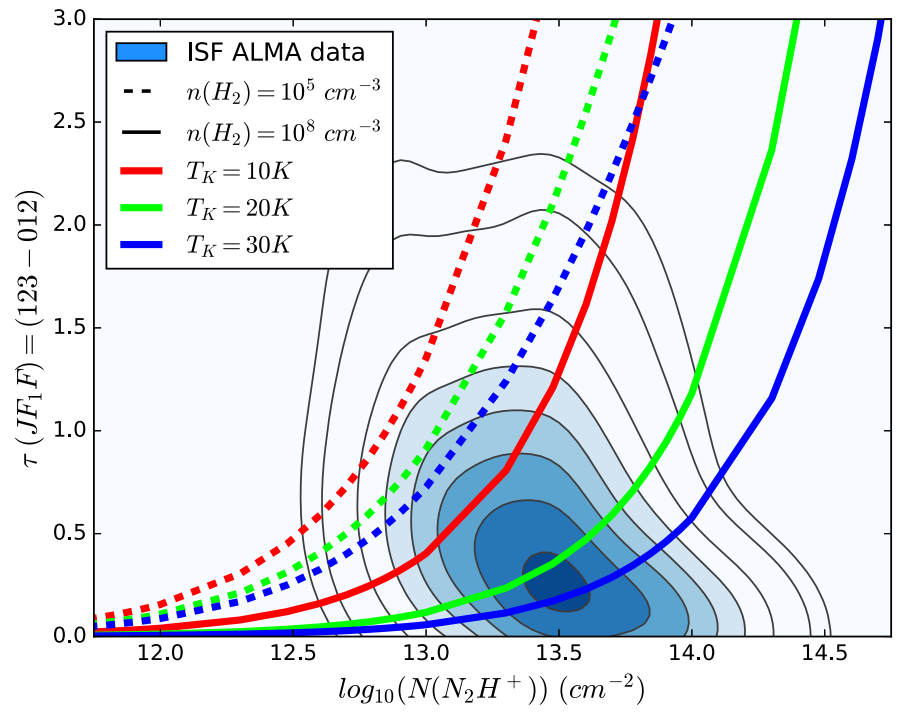

Fig. B.4. Distribution of the derived $\tau\left(J F_{1} F\right)=(123-012)$ opacities as function of the estimated total $\mathrm{N}_{2} \mathrm{H}^{+}$column density in the ISF. The plot represents the kernel density estimates of $>25000$ individual components extracted from our ALMA observations. The last contour encloses $95 \%$ of the components fitted in our spectra. For comparison, we overplot the different RADEX predictions for an isothermal gas at kinetic temperatures of $10 \mathrm{~K}$ (red), $20 \mathrm{~K}$ (green), and $30 \mathrm{~K}$ (blue) at densities $n\left(\mathrm{H}_{2}\right)$ between $10^{5} \mathrm{~cm}^{-3}$ (dashed lines) and $10^{8} \mathrm{~cm}^{-3}$ (solid lines), all calculated assuming a constant linewidth of $\Delta V=0.7 \mathrm{~km} \mathrm{~s}^{-1}$.

selective) increase of $\sim 40 \%$ on the total gas masses including opacity effects (e.g., Sect. 3.3). The large fitting uncertainties in heavily opaque lines prevent a more detailed treatment of these opacity effects. Still, their expected variations are within 


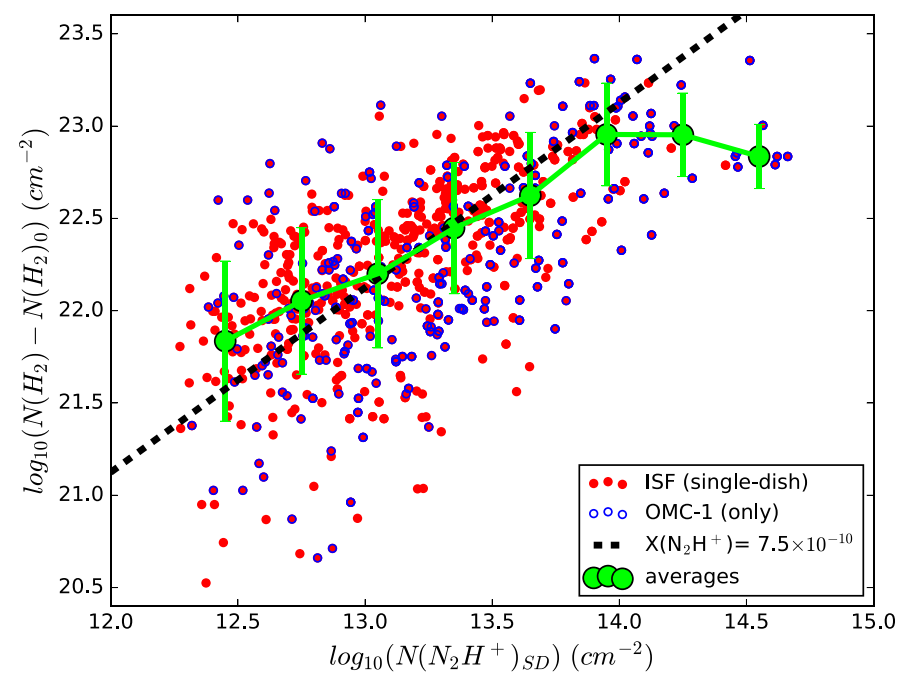

Fig. C.1. Derived total $\mathrm{N}_{2} \mathrm{H}^{+}$column density in our single-dish observations $\left(N\left(\mathrm{~N}_{2} \mathrm{H}^{+}\right)_{S D}\right)$ as function of the (normalized) Herschel $\mathrm{H}_{2}$ column density $\left(\mathrm{N}\left(\mathrm{H}_{2}\right)-N\left(\mathrm{H}_{2}\right)_{0}\right)$ for all the positions shown in Fig. 7 along the ISF (red) (see text for further details). The positions in OMC-1 are highlighted in blue. Both mean (points) and 1- $\sigma$ values (error bars) in bins of 0.3 dex are displayed in green. The expected correlation for a constant abundance ratio of $X\left(\mathrm{~N}_{2} \mathrm{H}^{+}\right)=7.5 \times 10^{-10}$ is indicated by a dashed black line.

the uncertainties already associated to our mass conversion factor estimated of $\sim 2-3$ (see Eq. (2)) and are not considered in our analysis.

\section{Appendix C: Single-dish $\mathrm{N}_{2} \mathrm{H}^{+}$abundances}

The good correlation found between the observed $\mathrm{N}_{2} \mathrm{H}^{+}$intensities and total $\mathrm{H}_{2}$ column densities derived in Fig. 7 suggests that this molecule may only exhibit small abundance variations across the entire ISF region. In the absence of a direct calibration of the $\mathrm{H}_{2}$ masses at the ALMA resolution, it is important to test the validity of this conclusion at the resolution of our single-dish observations.

In Fig. C.1, we display the estimated total $\mathrm{N}_{2} \mathrm{H}^{+}$column densities detected in our single-dish maps $\left(N\left(\mathrm{~N}_{2} \mathrm{H}^{+}\right)_{S D}\right)$ in all positions shown in Fig. 7 with at least one line component with $S / N \geq 3$. These $N\left(\mathrm{~N}_{2} \mathrm{H}^{+}\right)_{S D}$ values are derived following the approach presented by (Caselli et al. 2002, see also Appendix B.3). If needed, the contribution of several lines are added in spectra with multiple components. Each $N\left(\mathrm{~N}_{2} \mathrm{H}^{+}\right)_{S D}$ measurement is compared with the corresponding total gas column density $\mathrm{N}\left(\mathrm{H}_{2}\right)$ provided by previous Herschel measurements at the same position after the subtraction of the fit intercept (zero-level) derived in Eq. (2) (i.e. $\left.\mathrm{N}\left(\mathrm{H}_{2}\right)_{0}=1.7 \times 10^{22} \mathrm{~cm}^{-2}\right)$. For $\log _{10}\left(N\left(\mathrm{~N}_{2} \mathrm{H}^{+}\right)\right) \sim[12.5,14.0]$ and $\log _{10}\left(N\left(\mathrm{H}_{2}\right)-N\left(\mathrm{H}_{2}\right)_{0}\right) \sim[21.0,23.0]$ values, $70 \%$ of the respective column densities in OMC-2 are consistent, within a factor of two, with a constant abundance $X\left(\mathrm{~N}_{2} \mathrm{H}^{+}\right)=7.5 \times$ $10^{-10}$. The same relation is followed by the positions observed in OMC-1 despite their larger uncertainties in their molecular and dust emission properties. A systematic deviation towards higher abundances is observed at $\log _{10}\left(N\left(\mathrm{~N}_{2} \mathrm{H}^{+}\right)\right)>14.0$ and $\log _{10}\left(N\left(\mathrm{H}_{2}\right)-N\left(\mathrm{H}_{2}\right)_{0}\right) \sim 23.0$, although the low number of points at these high column densities prevents any further analysis. Exceptions of this well-behaved correlation are also found at higher resolutions in the vicinity $\left(\lesssim 20^{\prime \prime}\right)$ of Orion BN/KL hot core with almost no $\mathrm{N}_{2} \mathrm{H}^{+}$(1-0) emission in comparison with the detection of warm $\mathrm{NH}_{3}$ gas (e.g., Goddi et al. 2011). With a little influence in our global analysis though, the local interpretation of our ALMA observations in this particular region should be then taken with caution.

According to our single-dish results, $\mathrm{N}_{2} \mathrm{H}^{+}$is found at relatively constant abundance at the typical gas column densities traced in the ISF (see also Fig. B.4). Moreover, its absolute value appears to vary less than $50 \%$ compared to other filaments in clouds like Taurus (with $X\left(\mathrm{~N}_{2} \mathrm{H}^{+}\right)=5 \times 10^{-10}$; Tafalla \& Hacar 2015). The above single-dish $\mathrm{N}_{2} \mathrm{H}^{+}$abundances are also consistent with the results obtained from the comparison of SCUBA-derived $\mathrm{H}_{2}$ column densities (assuming the same $\mathrm{T}_{\text {dust }}$ values derived by Lombardi et al. 2014) and our new $\mathrm{N}_{2} \mathrm{H}^{+}$ ALMA observations. However, the different resolutions of both single-dish (with $\theta_{m b}$ between $36^{\prime \prime}$ and 14") and interferometric maps maps $\left(\theta_{m b}=4.5^{\prime \prime}\right)$ as well as the large uncertainties associated to these calculations (e.g., dust emissivity, filtering effects, etc.) prevent any further analysis. Still, we remark here that the total $\mathrm{H}_{2}$ masses in our maps are directly derived from the empirical relation between the observed $\mathrm{N}_{2} \mathrm{H}^{+}$intensities and Herschel column densities defined by Eq. (2) avoiding the assumption of any particular $\mathrm{N}_{2} \mathrm{H}^{+}$abundance. Although not directly involved in these latter estimates, the relatively stable $\mathrm{N}_{2} \mathrm{H}^{+}$abundance in regions like OMC-1 and OMC-2 support the use of the integrated intensity of this molecule as a reliable proxy of the total gas column densities within the wide range of physical conditions considered in this study. 